\title{
JAMES MAPS, SEGAL MAPS, AND THE KAHN-PRIDDY THEOREM
}

BY

\author{
J. CARUSO, F. R. COHEN, J. P. MAY AND L. R. TAYLOR
}

ABSTRACT. The standard combinatorial approximation $C\left(R^{n}, X\right)$ to $\Omega^{n} \Sigma^{n} X$ is a filtered space with easily understood filtration quotients $D_{q}\left(R^{n}, X\right)$. Stably, $C\left(R^{n}, X\right)$ splits as the wedge of the $D_{q}\left(R^{n}, X\right)$. We here analyze the multiplicative properties of the James maps which give rise to the splitting and of various related combinatorially derived maps between iterated loop spaces. The target of the total James map

$$
j=\left(j_{q}\right): \Omega^{n} \Sigma^{n} X \rightarrow \underset{q \geqslant 0}{X} \Omega^{2 n q} \Sigma^{2 n q} D_{q}\left(R^{n}, X\right)
$$

is a ring space, and $j$ is an exponential $H$-map. There is a total Segal map

$$
s=\underset{q \geqslant 0}{\chi} s_{q}: \underset{q \geqslant 0}{\chi} \Omega^{2 n q} \Sigma^{2 n q} D_{q}\left(R^{n}, X\right) \rightarrow \underset{q \geqslant 0}{\chi} \Omega^{3 n q} \Sigma^{3 n q} X^{[q]}
$$

which is a ring map between ring spaces. There is a total partial power map

$$
k=\left(k_{q}\right): \Omega^{n} \Sigma^{n} X \rightarrow \underset{q \geqslant 0}{\chi} \Omega^{n q} \Sigma^{n q} X^{[q]}
$$

which is an exponential $H$-map. There is a noncommutative binomial theorem for the computation of the smash power $\Omega^{n} \Sigma^{n} X \rightarrow \Omega^{n q} \Sigma^{n q} X^{[q]}$ in terms of the $k_{m}$ for $m \leqslant q$. The composite of $s$ and $j$ agrees with the composite of $k$ and the natural inclusion

$$
\underset{q \geqslant 0}{X} \Omega^{n q} \Sigma^{n q} X^{[q]} \rightarrow \underset{q \geqslant 0}{\chi} \Omega^{3 n q} \Sigma^{3 n q} X^{[q]} .
$$

This analysis applies to essentially arbitrary spaces $X$. When specialized to $X=S^{0}$, it implies an unstable version of the Kahn-Priddy theorem. The exponential property of the James maps leads to an analysis of the behavior of loop addition with respect to the stable splitting of $\Omega^{n} \Sigma^{n} X$ when $X$ is connected, and there is an analogous analysis relating loop addition to the stable splitting of $Q\left(X^{+}\right)$.

Our main purpose here is to analyze the algebraic properties of various combinatorially defined maps relating iterated loop spaces. The "James maps" referred to in the title will be extensions of the generalized James maps which we introduced in [6]. The "Segal maps" will be generalizations to the context of [6] of certain maps introduced by Segal [21] in his intriguing paper on the Kahn-Priddy theorem. Other families of combinatorial maps will also make their appearance. One application will

Received by the editors January 18, 1983.

1980 Mathematics Subject Classification. Primary 55P35, 55P47, 55Q05; Secondary 18F25, 55Q25, $55 \mathrm{~S} 15$.

Key words and phrases. James maps, Kahn-Priddy theorem, iterated loop space, splitting theorem, coefficient system, group completion. 
be the derivation of an unstable Kahn-Priddy theorem. Other applications have appeared in [8, 18 and 23$]$.

We shall prove the following five theorems. In the first three, $X$ is a based space such that $\pi_{0}(X)$ is countable. In all of them, $A$ is a CW complex which is assumed to be finite unless $X$ is (path) connected. We write $[A, X]$ for the set of based homotopy classes of based maps $A \rightarrow X$. Throughout, spaces are to be compactly generated, weak Hausdorff, and of the homotopy type of CW complexes; basepoints are to be nondegenerate.

To fix notation, recall from [6] that

$$
D_{q}(Y, X)=F(Y, q)^{+} \wedge_{\Sigma_{q}} X^{[q]}
$$

Here $X^{[q]}$ is the $q$ th smash power of $X, F(Y, q)$ is the configuration space of $q$-tuples of distinct points of $Y, Z^{+}$is the union of $Z$ with a disjoint basepoint, and $\Sigma_{q}$ is the $q$ th symmetric group with its natural permutation action on $F(Y, q)$ and $X^{[q]}$. By convention, $X^{[0]}=D_{0}(Y, X)=S^{0}$. If $Y$ is contractible, then $D_{1}(Y, X)$ is homotopy equivalent to $X$.

The spaces $D_{q}\left(R^{n}, X\right)$ are the basic building blocks for $\Omega^{n} \Sigma^{n} X$ and the subjects of our first two theorems. We specified a free action of $\Sigma_{q}$ on $F\left(R^{n}-0, q-1\right)$ in $[6,5.7]$ and we let $t(n, q)$ be 1 plus the embedding dimension of

$$
R^{n-1} \times F\left(R^{n}-0, q-1\right) / \Sigma_{q} .
$$

While these numbers give good destabilization for the generalized James maps of the following theorem, the reader need only think of them as some appropriate numbers between $n q$ and $2 n q$. Note that $t(n, 1)=n$.

THEOREM A. For $n \geqslant 2$, there exist natural maps

$$
j_{q}: \Omega^{n} \Sigma^{n} X \rightarrow \Omega^{t(n, q)} \Sigma^{t(n, q)} D_{q}\left(R^{n}, X\right)
$$

which satisfy the following properties:

(1) $j_{0}$ is constant at the point $1 \in S^{0}=D_{0}\left(R^{n}, X\right)$.

(2) $j_{1}$ is homotopic to the identity via $D_{1}\left(R^{n}, X\right) \simeq X$.

(3) $j_{r}(\alpha+\beta)=\sum_{p+q=r} j_{p}(\alpha) j_{q}(\beta)$ for $\alpha, \beta \in\left[A, \Omega^{n} \Sigma^{n} X\right]$.

For $0 \leqslant m \leqslant n$, let $\eta[m, n]$ denote the natural inclusion of $\Omega^{m} \sum^{m} X$ in $\Omega^{n} \Sigma^{n} X$; it induces the $(n-m)$-fold suspension on homotopy groups. In part (3), we have continued to write $j_{q}$ for the evident composite

$$
\Omega^{n} \Sigma^{n} X \rightarrow \Omega^{t(n, q)} \Sigma^{t(n, q)} D_{q}\left(R^{n}, X\right) \rightarrow \Omega^{2 n q} \Sigma^{2 n q} D_{q}\left(R^{n}, X\right) .
$$

The sums in (3) are induced by the usual loop space addition. The products are induced by the composite of the usual loop space smash product and application of the functor $\Omega^{2 n r} \Sigma^{2 n r}$ to certain canonical pairings

$$
D_{p}\left(R^{n}, X\right) \wedge D_{q}\left(R^{n}, X\right) \rightarrow D_{r}\left(R^{n}, X\right) .
$$

Sums and products have similar interpretations in our remaining theorems. 
Our second theorem introduces the Segal maps, and we need some notation in order to state their multiplicative properties. Observe that any homeomorphism $\sigma$ : $S^{r} \rightarrow S^{r}$ induces the conjugation homeomorphism $\tilde{\sigma}: \Omega^{r} \Sigma^{r} X \rightarrow \Omega^{r} \Sigma^{r} X$ specified by $\tilde{\sigma}(f)=\left(1 \wedge \sigma^{-1}\right) \circ f \circ \sigma$ on maps $f: S^{r} \rightarrow X \wedge S^{r}$. For $\sigma \in \Sigma_{r}$, let $\hat{\sigma}: \Omega^{r} \Sigma^{r} X^{[r]} \rightarrow$ $\Omega^{r} \Sigma^{r} X^{[r]}$ denote the composite $\left(\Omega^{r} \Sigma^{r} \sigma^{-1}\right) \circ \tilde{\sigma}$, so that $\hat{\sigma}(f)=\left(\sigma^{-1} \wedge \sigma^{-1}\right) \circ f \circ \sigma$. Let $S_{p, q} \subset \Sigma_{p+q}$ denote the set of $(p, q)$-shuffles, namely those permutations $\sigma$ such that $\sigma(i)<\sigma(j)$ whenever $1 \leqslant i<j \leqslant p$ or $p+1 \leqslant i<j \leqslant p+q$.

THEOREM B. For $n \geqslant 2$, there exist natural maps

$$
s_{q}: D_{q}\left(R^{n}, X\right) \rightarrow \Omega^{n q} \Sigma^{n q} X^{[q]}
$$

which satisfy the following properties:

(1) $s_{0}$ is the identity map of $S^{0}$.

(2) $s_{1}$ is homotopic to $\eta[0, n]$ via $D_{1}\left(R^{n}, X\right) \simeq X$.

(3) $\left(s_{q} \circ \Delta_{b}\right)(\alpha)=q ! \eta[0, n q] \Delta(\alpha)$ for $\alpha \in[A, X]$, where $\Delta: X \rightarrow X^{[q]}$ is the diagonal map and $\Delta_{b}: X \rightarrow D_{q}\left(R^{n}, X\right)$ is induced from $\Delta$ by $\Delta_{b}(x)=[b ; \Delta(x)]$ for any chosen $b \in F\left(R^{n}, q\right)$.

(4) $s_{p+q}(\alpha \beta)=\Sigma_{\sigma \in S_{p, q}} \hat{\sigma}\left(s_{p}(\alpha) s_{q}(\beta)\right)$ for $\alpha \in\left[A, D_{p}\left(R^{n}, X\right)\right]$ and $\beta \in$ $\left[A, D_{q}\left(R^{n}, X\right)\right], \alpha \beta$ being defined by means of the pairing $(*)$.

Recall that a map $X \rightarrow \Omega^{t} X^{\prime}$ extends uniquely to a $t$-fold loop map $\Omega^{t} \Sigma^{t} X \rightarrow \Omega^{t} X^{\prime}$. We shall also write $s_{q}$ for the $t$-fold loop map

$$
\Omega^{t} \Sigma^{t} D_{q}\left(R^{n}, X\right) \rightarrow \Omega^{n q+t} \Sigma^{n q+t} X^{[q]}
$$

whose restriction to $D_{q}\left(R^{n}, X\right)$ is the evident composite

$$
D_{q}\left(R^{n}, X\right) \rightarrow \Omega^{n q} \sum^{n q} X^{[q]} \rightarrow \Omega^{n q+t} \sum^{n q+t} X^{[q]} .
$$

With $t=t(n, q)$, this map $s_{q}$ can be composed with $j_{q}$. To study the composite, we introduce a map $k_{q}$ which we call the $q$ th partial power because of its relationship to the $q$ th smash power. We need further notation to describe this relationship. For $1 \leqslant m \leqslant q$, let $P_{m, q}$ denote the set of all partitions of $\{1,2, \ldots, q\}$ into $m$ disjoint nonempty subsets and let $c_{m, q}$ denote the cardinality of $P_{m, q}$. Define a partial order on the set of subsets of $\{1,2, \ldots, q\}$ by $s<t$ if the smallest element of $s$ is smaller than the smallest element of $t$. Then a partition $\rho \in P_{m, q}$ can be written as $\rho=\left\{s_{1}, \ldots, s_{m}\right\}$ with $s_{1}<\cdots<s_{m}$, and we define a corresponding map $\rho: X^{[m]} \rightarrow$ $X^{[q]}$ by

$$
\rho\left(x_{1}, \ldots, x_{m}\right)=\left(y_{1}, \ldots, y_{q}\right), \quad \text { where } y_{j}=x_{i} \text { if } j \in s_{i} .
$$

Thus $\rho$ is a composite of diagonal maps and permutations.

TheOREM C. For $n \geqslant 2$, there exist natural maps

$$
k_{q}: \Omega^{n} \Sigma^{n} X \rightarrow \Omega^{n q} \Sigma^{n q} X^{[q]}
$$

which satisfy the following properties, where $\alpha, \beta \in\left[A, \Omega^{n} \Sigma^{n} X\right]$ :

(1) $k_{0}$ is constant at the point $1 \in S^{0}=X^{[0]}$.

(2) $k_{1}$ is the identity map.

(3) $\eta[n q, n q+t(n, q)] k_{q}(\alpha)=\left(s_{q}^{\circ} j_{q}\right)(\alpha)$. 
(4) $k_{r}(\alpha+\beta)=\Sigma_{p+q=r} \Sigma_{\sigma \in S_{p . q}} \hat{\sigma}\left(k_{p}(\alpha) k_{q}(\beta)\right)$.

(5) $\alpha^{q}=\sum_{m=1}^{q} \sum_{\rho \in P_{m, q}}\left(\eta[n m, n q] \circ \Omega^{n m} \sum^{n m} \rho \circ k_{m}\right)(\alpha)$.

The last formula may look peculiar; it would remain correct for any choice of ordering of sets with $\{i\}<\{j\}$ if $i<j$. Since the $q$ th summand is just $k_{q}(\alpha)$, it gives an inductive calculation of all $k_{q}(\alpha)$ in terms of smash powers. While all this structure will surely prove useful in general, the obvious application is in the case $X=S^{0}$. Here the maps $\rho$ are all the identity, hence $\alpha^{q}=\sum_{m=1}^{q} c_{m, q} \eta[n m, n q] k_{m}(\alpha)$. This makes it a simple matter to derive explicit formulae for the $k_{q}$.

THEOREM D. For $n \geqslant 2$ and $\alpha \in\left[A^{+}, \Omega^{n} S^{n}\right]$,

$$
k_{q}(\alpha)=\alpha(\alpha-1) \cdots(\alpha-q+1),
$$

where $r \in\left[A^{+}, \Omega^{n} S^{n}\right]$ maps all of $A$ to a map $S^{n} \rightarrow S^{n}$ of degree $r$. If $A$ is a suspension and $\alpha(A)$ is contained in the component $\Omega_{0}^{n} S^{n}$ of degree-zero maps, then

$$
k_{q}(\alpha)=(-1)^{q-1}(q-1) ! \eta[n, n q](\alpha) .
$$

Here the second statement follows immediately from the first since the reduced diagonal $A \rightarrow A \wedge A$ is null homotopic and thus products are zero when $A$ is a suspension.

As Segal observed, the Kahn-Priddy theorem follows. For an unstable version, let us first fix some notations. For a based space $X$, we have an equivalence $\Sigma^{n}\left(X^{+}\right) \simeq$ $\Sigma^{n} X \vee S^{n}$. One form of the Hilton-Milnor theorem asserts that, for based spaces $U$ and $V$ with $V$ (path) connected,

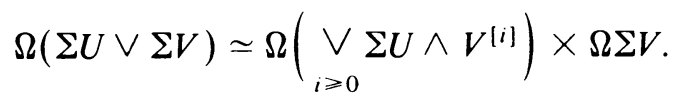

Taking $U=\Sigma^{t-1} X, V=S^{t-1}$ and looping $t-1$ times, $t \geqslant 2$, we obtain

$$
\Omega^{t} \Sigma^{t}\left(X^{+}\right) \simeq \Omega^{t} \mathcal{E}^{t} X \times \Omega^{t} S^{t}, \text { where } \mathcal{E}^{t} X=\underset{i \geqslant 0}{\bigvee} \Sigma^{t+(t-1) i} X .
$$

Let $B(Y, q)$ denote the generalized braid space $F(Y, q) / \Sigma_{q}$. It is clear that $D_{q}\left(Y, S^{0}\right)=B(Y, q)^{+}$. Thus $j_{q}: \Omega^{n} S^{n} \rightarrow \Omega^{t} \Sigma^{t} D_{q}\left(R^{n}, S^{0}\right), t=t(n, q)$, splits up to homotopy as $j_{q}^{\prime} \times j_{q}^{\prime \prime}$, where the maps

$$
j_{q}^{\prime}: \Omega^{n} S^{n} \rightarrow \Omega^{t} \mathcal{E}^{t} B\left(R^{n}, q\right) \text { and } j_{q}^{\prime \prime}: \Omega^{n} S^{n} \rightarrow \Omega^{t} S^{t}
$$

are defined once we fix a basepoint $b \in B\left(R^{n}, q\right)$.

Theorem E. For $n \geqslant 2, t=t(n, q)$, and $\alpha \in\left[A^{+}, \Omega^{n} S^{n}\right]$, where $A$ is a suspension and $\alpha(A) \subset \Omega_{0}^{n} S^{n}$,

$$
\left(s_{q} \circ j_{q}^{\prime}\right)(\alpha)=(-1)^{q-1}(q-1) ! \eta[n, n q+t](\alpha)-q ! \eta[t, n q+t] j_{q}^{\prime \prime}(\alpha) .
$$

Thus, if $p$ is a prime, then $\left(s_{p} \circ j_{p}^{\prime}\right)(\alpha) \equiv-\eta[n, n q+t](\alpha) \bmod p$.

Proof. Part (3) of Theorem B implies $s_{q}(\beta)=q ! \eta[t, n q+t](\beta)$ for any $\beta$ in the image of $\left[A^{+}, \Omega^{t} S^{t}\right]$ in $\left[A^{+}, \Omega^{t} \Sigma^{t} D_{q}\left(R^{n}, S^{0}\right)\right]$; hence the conclusion follows immediately from part (3) of Theorem $\mathrm{C}$ and the second formula of Theorem $\mathrm{D}$. 
All of our constructions pass to colimits over $n$, and $B\left(R^{\infty}, q\right)$ is equivalent to the classifying space $B \Sigma_{q}$. As usual, let $Q X=$ colim $\Omega^{n} \Sigma^{n} X$ and let $Q_{0} X$ be its basepoint component. We have a (based) equivalence $Q\left(B \Sigma_{q}^{+}\right) \simeq Q B \Sigma_{q} \times Q S^{0}$, and we write $j_{q}^{\prime}$ for the first coordinate of the restriction of $j_{q}$ to $Q_{0} S^{0}$ and $s_{q}^{\prime}$ for the map $Q B \Sigma_{q} \rightarrow Q_{0} S^{0}$ obtained by restriction of $s_{q}$. Clearly Theorem E implies that $s_{p}^{\prime} \circ j_{p}^{\prime}$ induces an isomorphism on the $p$-torsion in $\pi_{*} Q_{0} S^{0}$.

THEOREM F (KAHN-PRIDDY). For a prime $p$, the composite

$$
Q_{0} S^{0} \stackrel{j_{p}^{\prime}}{\rightarrow} Q B \Sigma_{p} \stackrel{s_{p}^{\prime}}{\rightarrow} Q_{0} S^{0}
$$

is a p-local equivalence.

The reader is invited to consult [18, pp. 61-66] for an outline of proofs and an application of the ideas above to the study of 2-primary exponents of homotopy groups of spheres. However, Theorems $\mathrm{B}$ and $\mathrm{C}$ show that some formulas on $[\mathbf{1 8}, \mathrm{p}$. 64] are incorrect.

REMARKS. (i) Kuhn [12] has proven that $j_{p}^{\prime}$ is not a second loop map. We do not know whether or not $j_{p}^{\prime}$ is homotopic to the map ( $t$ on $[10$, p. 110]) used by Kahn and Priddy.

(ii) Theorem B gives a map $s_{p}: B \Sigma_{p} \rightarrow Q S^{0}$ which lands in the component of maps of degree $p$ !, and $s_{p}^{\prime}$ is the infinite loop map adjoint to the translate of $s_{p}$ to $Q_{0} S^{0}$. The action of the infinite little cubes operad on $Q S^{0}$ leads to a map $\phi_{p}: B \Sigma_{p} \rightarrow Q S^{0}$ which lands in the component of maps of degree $p$, and Kahn and Priddy used the infinite loop map $\phi_{p}^{\prime}$ adjoint to the translate of $\phi_{p}$ to $Q_{0} S^{0}$. By Adams [1], $\phi_{p}^{\prime}$ and $s_{p}^{\prime}$ differ at most by an infinite loop self-equivalence of $Q B \Sigma_{p}$. They will be homotopic if and only if the two specified translates $B \Sigma_{p} \rightarrow Q_{0} S^{0}$ are homotopic; we suspect that this is true if $p=2$ but false if $p>2$.

(iii) Löffler and Ray [14] have very recently given a quick geometric proof of the Kahn-Priddy theorem.

(iv) In a slightly different direction, the second author, Peterson, and Selick have recently shown that any map $g: Q \Sigma R P^{\infty} \rightarrow Q S^{1}$ which induces an isomorphism on $\pi_{2}$ induces a split epimorphism on the 2-primary component of all higher homotopy groups; $g$ need not be an infinite loop map or even an $H$-map.

We shall derive several other results related to Theorem A. Let $P^{n}(p)$ denote the $\bmod p$ Moore space $S^{n-1} \cup_{p} e^{n}$. In [8], we used the $p$-local $H$-map $\Omega^{2} S^{3} \rightarrow$ $Q P^{2 p-1}(p)$ implied by the following result to obtain a simple proof of Mahowald's theorem [15] that $K(Z, 0)$ is a Thom spectrum.

TheOREM G. When localized at an odd prime $p$,

$$
j_{p}: \Omega^{2 n} S^{2 n+2 k-1} \rightarrow \Omega^{t(2 n, p)} \Sigma^{t(2 n, p)} D_{p}\left(R^{2 n}, S^{2 k-1}\right)
$$

becomes an H-map. Moreover, p-locally, $D_{p}\left(R^{2}, S^{2 k-1}\right) \simeq P^{2 p k-1}(p)$.

Although we have taken pains to make this paper reasonably self-contained, it is a sequel to [6]. We gave general splitting theorems there, and a major concern here is the analysis of their multiplicative behavior. In particular, we gave a new proof of 
Snaith's stable equivalence [22] between $\Omega^{n} \Sigma^{n} X$ and $\bigvee_{q \geqslant 1} D_{q}\left(R^{n}, X\right)$ for connected spaces $X$, and we shall prove that our splitting is compatible with products. Let $\Sigma^{\infty}$ denote the suspension functor from spaces to spectra (denoted $Q_{\infty}$ in [6]).

THEOREM H. For $n \geqslant 1$ and connected spaces $X$, the following is a natural commutative diagram in the stable category in which the horizontal arrows are equivalences:

$$
\begin{gathered}
\Sigma^{\infty}\left(\Omega^{n} \Sigma^{n} X \times \Omega^{n} \Sigma^{n} X\right) \stackrel{\Sigma_{r>1} \Sigma_{p+q=r} j_{p} \wedge_{q}}{\longrightarrow} \underset{r \geqslant 1}{\vee} \underset{p+q=r}{\vee} \Sigma^{\infty}\left(D_{p}\left(R^{n}, X\right) \wedge D_{q}\left(R^{n}, X\right)\right) \\
\downarrow \\
\Sigma^{\infty} \Omega^{n} \Sigma^{n} X \underset{\Sigma_{r>1} j_{r}}{\longrightarrow} \underset{r \geqslant 1}{\vee} \Sigma^{\infty} D_{r}\left(R^{n}, X\right)
\end{gathered}
$$

Here the map on the left is loop addition and that on the right is given by the canonical pairings $(*)$ alluded to above.

This completes our summary of the main results. The proofs are all based on a certain natural diagram

$$
C\left(R^{n}, X\right) \stackrel{g_{n}}{\leftarrow} C_{n} X \stackrel{\alpha_{n}}{\rightarrow} \Omega^{n} \Sigma^{n} X,
$$

recalled in $\S 2$, in which the left space is filtered with successive quotients $D_{q}\left(R^{n}, X\right)$, $g_{n}$ is a homotopy equivalence, and $\alpha_{n}$ is a homotopy equivalence if $X$ is connected and a group completion in general. In the connected case, all of the proofs are purely combinatorial: we simply write down maps relating the $C\left(R^{n}, X\right)$, determine their algebraic properties, and translate to $\Omega^{n} \Sigma^{n} X$ via the equivalence.

To extend to nonconnected spaces, such as $S^{0}$, we need some general properties of group completions. This material is independent of the rest of the paper and of independent interest. For example, it gives a very quick way of defining products in algebraic $K$-theory. It is presented in $\$ 1$.

We introduce the combinatorial pairings that we are concerned with in $\$ 2$ and study the James maps in $§ \S 3-5$. In particular, these sections contain generalized homological and homotopical variants of Theorem $\mathrm{H}$ and the proofs of Theorems $\mathrm{A}$ and G. \$§6-9 contain the proofs of Theorems B-D. An appendix gives a bit of needed geometry.

In $\$ 10$, we change context slightly and study the multiplicative properties of the James maps we used in [7] to stably split such spaces as $B\left(\Sigma_{\infty} \int G\right)$ for a topological monoid $G$ and $Q_{0}\left(X^{+}\right)$for a connected space $X$. The results here play a central role in Snaith's proofs of stable splittings of $B U$ and $B S p$ [23].

1. Universal properties of group completions. For simplicity, we agree that all given $H$-spaces are to be homotopy associative and commutative in this section. An $H$-space $Y$ is said to be grouplike if $\pi_{0} Y$ is a group. By a simple check of homotopy groups and our $\mathrm{CW}$-homotopy type assumption, it follows that the shearing map, $s(x, y)=(x y, y)$, is a homotopy equivalence. Restricted to $\{*\} \times Y$, the first coordinate of a homotopy inverse of $s$ gives a map $\chi: Y \rightarrow Y$ which provides inverse elements up to homotopy. Thus, a grouplike $H$-space is an Abelian group up to 
homotopy. Choose a point $z$ in each component $[z]$ of $Y$ and let $Y_{0}$ be the basepoint component. Then the map $Y \rightarrow Y_{0} \times \pi_{0}(Y)$, specified by $y \rightarrow(y \chi(z),[z])$ for $y \in[z]$, is a homotopy equivalence of $H$-spaces. We have the following observation.

LEMMA 1.1. Let $f: X \rightarrow X^{\prime}$ induce an isomorphism on integral homology and let $Y$ be a grouplike $H$-space. Then $f_{*}:\left[X^{\prime}, Y\right] \rightarrow[X, Y]$ is an isomorphism.

Proof. We may assume that $Y$ is connected. A fixed choice for associating iterated products determines a retraction $M Y \rightarrow Y$, where $M Y$ is the James construction on $Y$, and $M Y$ is equivalent to $\Omega \Sigma Y$. Since $\Sigma f$ is an equivalence, the conclusion for $\Omega \Sigma Y$ is obvious by adjunction. This implies the conclusion for $Y$.

Let $X$ be an $H$-space. A group completion of $X$ is an $H$-map $g: X \rightarrow Y$, where $Y$ is grouplike, such that $\pi_{0} Y$ is the universal group associated to the monoid $\pi_{0} X$ and $H_{*} Y$ is the localization of the Pontryagin ring $H_{*} X$ at its multiplicative submonoid $\pi_{0} X$ for any commutative coefficient ring. It is easily seen that this condition holds for general rings if it holds for fields [17, 1.4]. Clearly $g$ is an equivalence if $X$ itself is grouplike.

We shall prove the following observation of Segal [21] (whose countability hypothesis will be explained in the proof). Recall that two maps $f, f^{\prime}: X \rightarrow Y$ are said to be weakly homotopic, written $f \stackrel{\text { w }}{\simeq} f^{\prime}$, if $f k \simeq f^{\prime} k$ for any $k: A \rightarrow X$ whose domain $A$ is a finite $\mathrm{CW}$-complex. By a simple exercise in the use of classical obstruction theory, the conclusion of Lemma 1.1 remains valid for weak homotopy classes of maps.

Proposition 1.2. Let $g: X \rightarrow Y$ be a group completion, where $\pi_{0} X$ contains a countable cofinal sequence. Then for any grouplike $H$-space $Z$ and weak $H$-map $f$ : $X \rightarrow Z$, there exists a weak $H$-map $\tilde{f}: Y \rightarrow Z$, unique up to weak homotopy, such that $\tilde{f g} \stackrel{\mathrm{w}}{\simeq}$.

This has the following conceptual interpretation.

COROLlaRY 1.3. On the category of finite $C W$-complexes $A$, the natural transformation $g_{*}:[A, X] \rightarrow[A, Y]$ is universal with respect to natural transformations of Abelian monoid valued functors from $[A, X]$ to Abelian group valued represented functors [ $A, Z]$.

We shall also need the following result. Both it and the recognition of its role in the present proof of the Kahn-Priddy theorem are due to the first author.

Proposition 1.4. Let $g: X \rightarrow Y$ and $g^{\prime}: X^{\prime} \rightarrow Y^{\prime}$ be group completions, where $\pi_{0} X$ and $\pi_{0} X^{\prime}$ contain countable cofinal sequences. Then for any grouplike $H$-space $Z$ and weakly homotopy bilinear map $f: X \wedge X^{\prime} \rightarrow Z$, there exists a weakly homotopy bilinear map $\tilde{f}: Y \wedge Y^{\prime} \rightarrow Z$, unique up to weak homotopy, such that $\tilde{f}\left(g \wedge g^{\prime}\right) \stackrel{\text { w }}{\simeq} f$.

By a (weak) $H$-semiring space we understand a space $X$ with two $H$-space structures which satisfy the axioms for a commutative ring, with the exception of the existence of additive inverses, up to (weak) homotopy. We require the additive unit to be a strict multiplicative zero, so that the multiplication factors through $X \wedge X$. 
We say that $X$ is a (weak) $H$-ring space if it is additively grouplike. We have the following consequence.

Corollary 1.5. Let $g: X \rightarrow Y$ be a group completion of the additive $H$-space structure of an $H$-semiring space $X$. Then $Y$ admits a unique structure of weak $H$-ring space such that $g$ is a map of weak H-semiring spaces.

Proof. With $f$ the composite of $g$ and the product $X \wedge X \rightarrow X, \tilde{f}: Y \wedge Y \rightarrow Y$ gives the product on $Y$. With unit $g(1)$, the unit laws for $Y$ follow from the uniqueness clause of Proposition 1.2. The associativity and commutativity of $\tilde{f}$ follow from the uniqueness clause of Proposition 1.4.

We digress to point out how this yields products in algebraic $K$-theory. With basepoint $0, \pi_{*} Y$ is clearly a graded commutative ring for any weak $H$-ring space $Y$.

COROLlaRY 1.6. For a commutative ring $R, K_{*} R$ is a graded commutative ring.

Proof. For quickness, we take $K_{0} R=Z$; the argument is easily modified to account for the usual $K_{0} R$. Let $B \mathcal{G L}(R)$ denote the disjoint union of the classifying spaces $B G L(n, R)$. On passage to classifying spaces, the standard sums and tensor products,

$$
\bigoplus: G L(m, R) \times G L(n, R) \rightarrow G L(m+n, R)
$$

and

$$
\otimes: G L(m, R) \times G L(n, R) \rightarrow G L(m n, R),
$$

give $B \mathcal{G L}(R)$ a structure of $H$-semiring space in which all associativity and unit laws hold strictly. Regarding $B \mathcal{G}(R)$ as a topological monoid under $\oplus$, we can define $K R=\Omega B(B \mathcal{G}(R))$. The natural map $\zeta: B \mathcal{G L}(R) \rightarrow K R$ is known to be a group completion, and this implies $K R \simeq B G L(R, \infty)^{+} \times Z$, where the + denotes the plus construction (see $[2, \S 3.2]$ for discussion). Thus $K_{*} R=\pi_{*} K R$. The previous corollary gives $K R$ a structure of weak $H$-ring space such that $\zeta$ is a map of weak $H$-semiring spaces.

In our applications of Proposition 1.4, we shall often have three sequences $X_{q}, Y_{q}$ and $Z_{q}, q \geqslant 0$, of additive $H$-spaces, with sums written $\oplus$. We shall also have homotopy bilinear pairings $\otimes: X_{p} \times Y_{q} \rightarrow Z_{p+q}$. Thus, we shall have composite maps

$$
\square: \underset{p+q=r}{X} X_{p} \times Y_{q} \stackrel{\times_{p+q=r} \otimes}{\rightarrow} \underset{p+q=r}{X} Z_{r} \stackrel{\oplus}{\rightarrow} Z_{r} .
$$

Sending $X_{p+q=r}\left(x_{p}, y_{q}\right)$ to the sequences beginning with $\left(x_{0}, \ldots, x_{r}\right)$ and $\left(y_{0}, \ldots, y_{r}\right)$ and ending with the units 0 of the $X_{s}$ and $Y_{s}$ for $s>r$, we obtain inclusions

$$
\underset{p+q=r}{X} X_{p} \times Y_{q} \subset\left(\underset{p \geqslant 0}{X} X_{p}\right) \times\left(\underset{q \geqslant 0}{X} Y_{q}\right) .
$$

On the other hand, we have an evident inclusion

$$
\left(\underset{p \geqslant 0}{X} X_{p}\right) \times\left(\underset{q \geqslant 0}{X} Y_{q}\right) \subset \underset{r \geqslant 0}{X}\left(\underset{p+q=r}{X} X_{p} \times Y_{q}\right) .
$$


Thus the maps $\square$ above determine and are determined by a single map

$$
\square:\left(\underset{p \geqslant 0}{X} X_{p}\right) \times\left(\underset{q \geqslant 0}{X} Y_{q}\right) \rightarrow \underset{r \geqslant 0}{X} Z_{r} .
$$

With $X_{q}=Y_{q}=Z_{q}$ and with $1 \in X_{0}$, it is evident how to specify homotopy unity, associativity, and commutativity conditions on $\otimes$ so as to ensure that $X=\times_{q \geqslant 0} X_{q}$ is an $H$-semiring space under $\square$, the additive $H$-space structure being the product of the sums $\oplus$ on the $X_{q}$. When these conditions hold, we let $U X \subset X$ be the unit space $\{1\} \times\left(X_{q \geqslant 1} X_{q}\right)$; it is an $H$-space under $\square$. With these notations, Proposition 1.4 gives the following result.

Corollary 1.7. Let $X=X_{q \geqslant 0} X_{q}$ be an H-semiring space as above and let $g_{q}$ : $X_{q} \rightarrow Y_{q}$ be group completions of the $X_{q}$. Then there are pairings $\otimes: Y_{p} \wedge Y_{q} \rightarrow Y_{p+q}$ which give $Y=X_{q \geqslant 0} Y_{q}$ a structure of weak $H$-ring space such that $g=X_{q \geqslant 0} g_{q}$ : $X \rightarrow Y$ is a map of weak $H$-semiring spaces. Moreover, $U Y$ is a grouplike weak $H$-space.

Proof. As in Corollary 1.5, the pairings are given by Proposition 1.4 and the desired algebraic properties are inherited by uniqueness. The last statement results from application of the following standard lemma to the Abelian groups $\pi_{0}\left(Y_{q}\right)$.

LEMMA 1.8. Suppose a sequence of Abelian groups $A_{r}, r \geqslant 0$, with a unital, associative, and commutative system of pairings $A_{p} \otimes A_{q} \rightarrow A_{p+q}$ is given. Then the set of formal sums $\Sigma_{r \geqslant 0} a_{r}$, with $a_{r} \in A_{r}$ and $a_{0}=1$, is a group with respect to the product

$$
\left(\sum_{p} a_{p}\right)\left(\sum_{p} b_{q}\right)=\sum_{r}\left(\sum_{p+q=r} a_{p} b_{q}\right) .
$$

PRoof. Regard finite sums $\sum_{r=0}^{s} a_{r}$ as formal sums with $a_{t}=0$ for $t>s$. Let $b_{0}=1$, assume inductively that $\sum_{r=0}^{s-1} a_{r}$ admits the inverse $b_{s-1}$, and set $b_{s}=$ $\Sigma_{r}(-1)^{r} b_{s-1}^{r+1} a_{s}^{r}$, where $a_{s}$ denotes the sequence with $s$ th term $a_{s}$ and remaining terms 0 . Then $b_{s}$ is inverse to $\sum_{r=0}^{s} a_{r}$ and, inductively, the components of $b_{s}$ in $A_{r}$, for $r<s$, are independent of $s$. The resulting limit element $b$ is inverse to $\Sigma_{r} a_{r}$.

Use of infinite products makes the following comment obligatory.

REMARK 1.9. Infinite products of $\mathrm{CW}$-homotopy types are not $\mathrm{CW}$-homotopy types, and we agree to replace them by weakly homotopy equivalent $\mathrm{CW}$-complexes without change of notation. By Milnor [19] for the functors $\Omega^{n}$, Lewis [13] for the functor $Q$, and [17, Appendix] for our combinatorial functors, all other constructions we use do preserve $\mathrm{CW}$-homotopy types.

The rest of the section is devoted to the proofs of Propositions 1.2 and 1.4, and we begin with the former.

Let $\left\{a_{i}\right\}$ be a cofinal sequence in $\pi_{0} X$. This means that $a_{i+1}=b_{i} a_{i}$ for some $b_{i}$ and that, for any $c \in \pi_{0} X$, there exists $d \in \pi_{0} X$ such that $d c=a_{i}$ for some $i$. Let $\bar{X}$ denote the telescope of the sequence of left translations $b_{i}: X \rightarrow X ; X$ is contained in $\bar{X}$ as the base of the telescope. Construct $\bar{Y}$ and $\bar{Z}$ similarly by use of the elements $g\left(b_{i}\right)$ and $f\left(b_{i}\right)$. Since $\pi_{0} Y$ and $\pi_{0} Z$ are groups, the translations here are all equivalences, hence so are the natural inclusions $Y \rightarrow \bar{Y}$ and $Z \rightarrow \bar{Z}$. Since $f$ and $g$ 
are weak $H$-maps, they commute up to weak homotopy with translations and so induce maps $\bar{f}: \bar{X} \rightarrow \bar{Z} \simeq Z$ and $\bar{g}: \bar{X} \rightarrow \bar{Y} \simeq Y$ whose restrictions to $X$ are $f$ and $g$. It is this use of telescopes which forces our use of weak homotopy, and the $\lim ^{1}$ exact sequence can often be used to obtain more precise conclusions.

It is an easy consequence of the group completion property and the standard description of localizations of rings as colimits (e.g. [17, p.63]) that $\bar{g}: \bar{X} \rightarrow Y$ induces an isomorphism on homology. By Lemma 1.1 , there results a map $\tilde{f}: Y \rightarrow Z$ such that $\tilde{f} \bar{g} \simeq \bar{f}$ and thus $\tilde{f} g \simeq f$.

To see that $\tilde{f}$ is a weak $H$-map, observe that $\bar{g} \times \bar{g}$ induces an isomorphism on homology. While $\bar{X}$ is not an $H$-space, in general, it is easy to see (using homotopy commutativity) that the product $\phi$ on $X$ extends to a product $\bar{\phi}$ on $\bar{X}$ which is compatible up to weak homotopy with the products $\phi$ on $Y$ and $Z$. Thus

$$
\phi(\tilde{f} \times \tilde{f})(\bar{g} \times \bar{g}) \simeq \phi(\bar{f} \times \bar{f}) \stackrel{\mathrm{w}}{\simeq} \bar{f} \bar{\phi} \simeq \tilde{f} g \bar{\phi} \stackrel{\mathrm{w}}{\simeq} \tilde{f} \phi(\bar{g} \times \bar{g})
$$

and therefore $\phi(\tilde{f} \times \tilde{f}) \stackrel{\mathrm{w}}{\simeq} \tilde{f} \phi$ by the weak homotopy version of Lemma 1.1.

Finally, suppose given a second weak $H$-map $k: Y \rightarrow Z$ such that $k g \stackrel{\text { w }}{\simeq} f$. Since $k$ is a weak $H$-map, we find easily that $k \bar{g} \stackrel{\text { w }}{\simeq} \bar{f} \simeq \tilde{f} \bar{g}$ and therefore $k \stackrel{\text { w }}{\simeq} \tilde{f}$ by Lemma 1.1 again.

We use function $H$-spaces to prove Proposition 1.4. For $H$-spaces $Y$ and $Z$, let $H(Y, Z)$ be the space of based weak $H$-maps $Y \rightarrow Z$. Then $H(Y, Z)$ is an $H$-space under the product induced by $Z,(j k)(y)=j(y) k(y)$. The homotopy commutativity of $Z$ ensures that $j k$ is again a weak $H$-map. The constant map at the unit of $Z$ is the unit of $H(Y, Z)$, and $H(Y, Z)$ is evidently homotopy associative and commutative. Moreover, if $Z$ is grouplike with a homotopy inverse map $\chi$, then $H(Y, Z)$ is grouplike with a homotopy inverse map specified by $j \rightarrow \chi \cdot j$.

We have the following interpretation of weak homotopy bilinearity.

LemmA 1.10. Let $X, Y$ and $Z$ be $H$-spaces. $A$ map $f: X \wedge Y \rightarrow Z$ is weakly homotopy bilinear if and only if its adjoint $\hat{f}$ provides a weak $H$-map $X \rightarrow H(Y, Z)$. The same conclusion holds with the roles of $X$ and $Y$ reversed.

Now adopt the notations of Proposition 1.4. The adjoint $X \rightarrow H\left(X^{\prime}, Z\right)$ of $f$ is a weak $H$-map, so extends uniquely to a weak $H$-map $Y \rightarrow H\left(X^{\prime}, Z\right)$ with adjoint $\bar{f}$ : $Y \wedge X^{\prime} \rightarrow Z$. Since $\bar{f}$ is weakly homotopy bilinear, its adjoint on the other side $X^{\prime} \rightarrow H(Y, Z)$ is a weak $H$-map, so extends uniquely to a weak $H$-map $Y^{\prime} \rightarrow H(Y, Z)$ with adjoint $\tilde{f}: Y \wedge Y^{\prime} \rightarrow Z$. The uniqueness clause of Proposition 1.2 implies the uniqueness of the weakly homotopy bilinear map $\tilde{f}$.

2. Combinatorial pairings; loop sums and smash products. As in [6], let $\Lambda$ be the category of finite based sets $\mathbf{r}=\{0,1, \ldots, r\}$ (with basepoint 0 ) and based injections. A coefficient system $C$ is a contravariant functor from $\Lambda$ to spaces with zeroth space $\bigodot_{0}$ a point. Associated to any coefficient system $\mathcal{C}$ and based space $X$ (with basepoint *) there is a space

$$
C X=C(\bigodot, X)=\coprod_{r \geqslant 0} \bigodot_{r} \times X^{r} /(\sim)
$$


where the equivalence relation is specified by $(c \phi, x) \sim(c, \phi x)$ for $\phi: \mathbf{r} \rightarrow \mathbf{s}$ in $\Lambda$, $c \in \mathcal{C}_{s}$, and $x=\left(x_{1}, \ldots, x_{r}\right) \in X^{r}$; here $\phi x=\left(x_{1}^{\prime}, \ldots, x_{s}^{\prime}\right)$, where $x_{\phi(i)}^{\prime}=x_{l}$ and $x_{j}^{\prime}=*$ if $j \notin \operatorname{Im} \phi$.

Write $[c ; x]$ for the image of $(c, x)$ in $C X$. Filter $C X$ by $F_{q} C X=\amalg_{j \leqslant q} e_{j} \times X^{j} /(\sim)$, give the $F_{q} C X$ the quotient topology, and give $C X$ the colimit topology. The inclusion of $F_{q-1} C X$ in $F_{q} C X$ is a cofibration with quotient

$$
F_{q} C X / F_{q-1} C X=D_{q}(e, X)=\bigodot_{q}^{+} \wedge_{\Sigma_{q}} X^{[q]} .
$$

Details of the above are in [6]. There we also talked about $\Pi$-spaces. At the price of considerable extra verbiage to keep track of pairings of $\Pi$-spaces, all of our combinatorial work could be carried out in the more general context.

For a space $Y$, let $e(Y)$ be the coefficient system whose $q$ th space is $F(Y, q)$. For $\phi: \mathbf{r} \rightarrow \mathbf{s}$ in $\Lambda$ and $\left\langle y_{1}, \ldots, y_{s}\right\rangle \in F(Y, s)$,

$$
\left\langle y_{1}, \ldots, y_{s}\right\rangle \phi=\left\langle y_{\phi(1)}, \ldots, y_{\phi(r)}\right\rangle \text {. }
$$

These are the most important examples for the technical part of our work. We write $C(Y, X)$ and $D_{q}(Y, X)$ for the resulting spaces. It should always be kept in mind that these are functors of $X$ but are only functorial with respect to injective maps of $Y$.

Other examples we need are operads, especially the little cubes operads $\bigodot_{n}$. The $q$ th space of $e_{n}$ consists of $q$-tuples of linear embeddings $J^{n} \rightarrow J^{n}$ with disjoint images, where $J$ is the open unit interval. Morphisms of $\Lambda$ act just as above. Here we write $C_{n} X$ and $D_{n, q} X$ for the resulting spaces. Via a homeomorphism $J \cong R$, we can replace $J$ by $R$. Then the map of coefficient systems $\bigodot_{n} \rightarrow C\left(R^{n}\right)$, obtained by sending cubes to their centers, induces natural homotopy equivalences

$$
g_{n}: C_{n} X \rightarrow C\left(R^{n}, X\right) \text { and } g_{n}: D_{n, q} X \rightarrow D_{q}\left(R^{n}, X\right) .
$$

We write $g_{n}^{-1}$ for any chosen homotopy inverses.

Regarding $S^{n}$ as $I^{n} / \partial I^{n}$, we obtain a natural map $\alpha_{n}: C_{n} X \rightarrow \Omega^{n} \Sigma^{n} X$ by the following simple prescription, where $s \in S^{n}$ :

$$
\alpha_{n}\left[\left\langle c_{1}, \ldots, c_{q}\right\rangle, x_{1}, \ldots, x_{q}\right](s)= \begin{cases}x_{j} \wedge t & \text { if } c_{j}(t)=s, \\ * & \text { if } s \notin \bigcup_{j} \operatorname{Im} c_{j} .\end{cases}
$$

If $X$ is connected, $\alpha_{n}$ is a homotopy equivalence. In general, $\alpha_{n}$ is a group completion. We set $\beta_{n}=\alpha_{n} g_{n}^{-1}$.

Proofs of the above statements may be found in [16,5, 20 and 4]; they need not concern us here. What does concern us is how these maps relate to various standard maps (loop sums, smash products, etc.) between loop spaces. As observed in [16], such comparisons are quite trivial verifications in view of the very simple and explicit nature of the maps $g_{n}$ and $\alpha_{n}$. To define the model level analogs of loop sums and smash products, and for other purposes, we first note that $\Lambda$ has sums and products, and then introduce notions of additive and multiplicative pairings of coefficient systems. 
Definitions 2.1. (i) Define the wedge sum $\vee: \Lambda \times \Lambda \rightarrow \Lambda$ by $\mathbf{p} \vee \mathbf{q}=\mathbf{p}+\mathbf{q}$ on objects and, for $\phi: \mathbf{p} \rightarrow \mathbf{p}^{\prime}$ and $\psi: \mathbf{q} \rightarrow \mathbf{q}^{\prime}$,

$$
(\phi \vee \psi)(i)= \begin{cases}\phi(i) & \text { if } 1 \leqslant i \leqslant p, \\ \psi(i-p)+p^{\prime} & \text { if } p<i \leqslant p+q .\end{cases}
$$

(ii) Define the smash product $\wedge: \Lambda \times \Lambda \rightarrow \Lambda$ by $\mathbf{p} \wedge \mathbf{q}=\mathbf{p q}$ on objects and, for $\phi: \mathbf{p} \rightarrow \mathbf{p}^{\prime}$ and $\psi: \mathbf{q} \rightarrow \mathbf{q}^{\prime}$,

$(\phi \wedge \psi)((i-1) q+j)=(\phi(i)-1) q^{\prime}+\psi(j)$, where $1 \leqslant i \leqslant p$ and $1 \leqslant j \leqslant q$.

Definitions 2.2. Let $Q, \mathscr{B}$ and $\mathcal{C}$ be coefficient systems.

(i) A sum $\bigoplus:(Q, \mathscr{B}) \rightarrow C$ is a collection of maps $\mathbb{Q}_{p} \times \mathscr{B}_{q} \rightarrow e_{p+q}$ which define a natural transformation from $Q \times \mathscr{B}$ to $e \circ V$.

(ii) A product $\otimes:(\mathscr{Q}, \mathscr{B}) \rightarrow C$ is a collection of maps $\mathbb{Q}_{p} \times \mathscr{B}_{q} \rightarrow \mathcal{C}_{p q}$ which define a natural transformation from $Q \times \Re$ to $\circlearrowright \circ \wedge$.

Morphisms of such pairings are triples of maps of coefficient systems which commute with the given natural transformations. Via the evident $r$-fold wedge sum and smash product on $\Lambda$, there are obvious generalizations to $r$-fold pairings.

The following lemmas are immediate from the definitions. We write $A X$ for $C(\mathbb{Q}, X)$, and so forth, to abbreviate notations.

Lemma 2.3. For a sum $\oplus:(\mathscr{Q}, \Re) \rightarrow \bigodot$ and based spaces $X$ and $Y$, there is a natural induced map $\oplus: A X \times B Y \rightarrow C(X \vee Y)$ specified on elements by the formula

$$
\left[a ; x_{1}, \ldots, x_{p}\right] \oplus\left[b ; y_{1}, \ldots, y_{q}\right]=\left[a \oplus b ; x_{1}, \ldots, x_{p}, y_{1}, \ldots, y_{q}\right] .
$$

This map carries $F_{p} \times F_{q}$ to $F_{p+q}$ and so passes to filtration quotients to give maps

$$
\bigoplus: D_{p}(\Theta, X) \wedge D_{q}(\Re, Y) \rightarrow D_{p+q}(\bigodot, X \vee Y)
$$

Often $X=Y$. We then continue to write $\oplus$ for the internal sums obtained by composing with $C(\nabla)$ or $D_{p+q}(1, \nabla)$, where $\nabla: X \vee X \rightarrow X$ is the folding map.

LEMma 2.4. For a product $\otimes:(\mathscr{Q}, \mathscr{B}) \rightarrow \bigodot$ and based spaces $X$ and $Y$, there is $a$ natural induced map $\otimes: A X \wedge B Y \rightarrow C(X \wedge Y)$ specified on elements by the formula

$$
\left[a ; x_{1}, \ldots, x_{p}\right] \otimes\left[b ; y_{1}, \ldots, y_{q}\right]=\left[a \otimes b ; \underset{(i, j)}{X} x_{i} \wedge y_{j}\right],
$$

where $1 \leqslant i \leqslant p, 1 \leqslant j \leqslant q$, and the set of pairs is ordered lexicographically. This map carries $F_{p} \wedge F_{q}$ to $F_{p q}$ and so passes to filtration quotients to give maps

$$
\otimes: D_{p}(Q ; X) \wedge D_{q}(\mathscr{B} ; Y) \rightarrow D_{p q}(\bigodot, X \wedge Y) \text {. }
$$

Often we will have a canonical map $f: X \wedge Y \rightarrow Z$, the choice of which should be clear from context. We then continue to write $\otimes$ for the products obtained by composing with $C(f)$ or $D_{p q}(1, f)$.

A word about basepoints and the case $p=0$ (or $q=0$ ) is in order. $X$ has basepoint $*$ and we define $X^{0}=\{*\}$. We denote adjoined disjoint basepoints by 0 and think of $S^{0}$ as $\{1\}^{+}$. We take $X^{[0]}=\{*\}^{+}$with basepoint 0 , not $*$. Since $\bigodot_{0}$ is also a point $*$, this forces the convention

$$
D_{0}(\bigodot, X)=e_{0}^{+} \wedge X^{[0]}=\left(\bigodot_{0} \times X^{0}\right)^{+}=S^{0}, \quad \text { with }(*, *)=1 .
$$


All maps in the lemmas are based, where the basepoint 0 is used in forming smash products when $p=0$ or $q=0$, and $1 \oplus[b ; y]=[b ; y]$ while $1 \otimes[b ; y]=1$.

EXAMPLE 2.5. For any spaces $Y$ and $Z$, we have a sum $\oplus:(\bigodot(Y), \varrho(Z)) \rightarrow$ C $(Y \amalg Z)$ specified by

$$
\left\langle y_{1}, \ldots, y_{p}\right\rangle+\left\langle z_{1}, \ldots, z_{q}\right\rangle=\left\langle y_{1}, \ldots, y_{p}, z_{1}, \ldots, z_{q}\right\rangle .
$$

If $i: Y \amalg Z \rightarrow W$ is an injective map, we can compose with $C(1, i)$ to obtain $\oplus$ : $(\bigodot(Y), \mathcal{C}(Z)) \rightarrow \mathcal{C}(W)$. When $Y=Z=W$, we say that an injection $i$ is good if its restrictions $Y \rightarrow Y$ are both homotopic through injections to the identity map. This is just enough to ensure that $C(Y, X)$ is an $H$-space under $\oplus$.

We have analogous $r$-fold sums defined for $r$-tuples of spaces and we have the analogous notion of a good injection $\amalg_{1 \leqslant i \leqslant r} Y \rightarrow Y$. Of course, if $Y$ is a manifold, such injections are embeddings and a homotopy through such embeddings defines an isotopy. We shall prove the following uniqueness result in the appendix.

LEMMA 2.6. For $n \geqslant 2$, any two good embeddings $\amalg_{1 \leqslant i \leqslant r} R^{n} \rightarrow R^{n}$ are isotopic.

EXAMPLE 2.7. For any operad $\mathcal{C}$ and any point $c \in \mathcal{C}_{2}$, the operad structure maps $\gamma(c): e_{p} \times e_{q} \rightarrow e_{p+q}$ specify a sum $\bigoplus:(e, C) \rightarrow C$. If $e_{1}$ is connected (as always holds in practice), then $C X$ is an $H$-space under $\oplus$. Similarly, any element of $\bigodot_{r}$ gives an $r$-fold sum on $e_{\text {; }}$ if $e_{r}$ is connected, any two such sums give homotopic $r$-fold sums on $C X$. When $\mathcal{C}$ is the little $n$-cubes operad and $c=\left\langle c_{1}, c_{2}\right\rangle, \gamma(c)$ is simply given by composition of little cubes:

$$
\gamma(c)\left(\left\langle a_{1}, \ldots, a_{p}\right\rangle,\left\langle b_{1}, \ldots, b_{q}\right\rangle\right)=\left\langle c_{1} a_{1}, \ldots, c_{1} a_{p}, c_{2} b_{1}, \ldots, c_{2} b_{q}\right\rangle .
$$

Via $J^{n} \cong R^{n},\left\langle c_{1}, c_{2}\right\rangle$ defines a good embedding $R^{n} \amalg R^{n} \rightarrow R^{n}$. It also defines a pinch map $S^{n} \rightarrow S^{n} \vee S^{n}$, namely the Pontryagin-Thom construction on this embedding, and thus a loop sum $*$ on $\Omega^{n} X$ for any $X$. With these choices, the following lemma is immediate.

LEMMA 2.8. The following diagram commutes for any $X$ :

$$
\begin{array}{ccccc}
C\left(R^{n}, X\right) \times C\left(R^{n}, X\right) & \stackrel{g_{n} \times g_{n}}{\leftarrow} & C_{n} X \times C_{n} X & \stackrel{\alpha_{n} \times \alpha_{n}}{\rightarrow} & \Omega^{n} \Sigma^{n} X \times \Omega^{n} \Sigma^{n} X \\
\oplus \downarrow & & \downarrow \oplus & & \downarrow * \\
C\left(R^{n}, X\right) & \stackrel{g_{n}}{\leftarrow} & C_{n} X & \stackrel{\alpha_{n}}{\rightarrow} & \Omega^{n} \Sigma^{n} X
\end{array}
$$

There are analogous examples of products.

EXAMPLE 2.9. For any spaces $Y$ and $Z$, we have a product $\otimes:(\bigodot(Y), \varrho(Z)) \rightarrow$ $\mathcal{C}(Y \times Z)$ specified by

$$
\left\langle y_{1}, \ldots, y_{p}\right\rangle \otimes\left\langle z_{1}, \ldots, z_{q}\right\rangle=\left\langle\underset{(i, j)}{X}\left(y_{i}, z_{j}\right)\right\rangle,
$$

where $1 \leqslant i \leqslant p, 1 \leqslant j \leqslant q$, and the pairs are ordered lexicographically. 
EXAMPLE 2.10. We have a product $\otimes:\left(\bigodot_{m}, \bigodot_{n}\right) \rightarrow \bigodot_{m+n}$ specified by

$$
\left\langle a_{1}, \ldots, a_{p}\right\rangle \otimes\left\langle b_{1}, \ldots, b_{q}\right\rangle=\left\langle\underset{(i, j)}{X} a_{i} \times b_{j}\right\rangle,
$$

where $a_{i} \times b_{j}: J^{m+n} \rightarrow J^{m+n}$ is the product little cube.

The following lemma is another immediate verification.

LEMMA 2.11. The following diagram commutes for any $X$ and $Y$ :

$$
\begin{array}{ccccc}
C\left(R^{m}, X\right) \wedge C\left(R^{n}, Y\right) & \stackrel{g_{m} \wedge g_{n}}{\leftarrow} & C_{m} X \wedge C_{n} Y & \stackrel{\alpha_{m} \wedge \alpha_{n}}{\rightarrow} & \Omega^{m} \Sigma^{m} X \wedge \Omega^{n} \Sigma^{n} Y \\
\otimes \downarrow & & \downarrow \otimes & & \downarrow \wedge \\
C\left(R^{m+n}, X \wedge Y\right) & \stackrel{g_{m+n}}{\leftarrow} & C_{m+n}(X \wedge Y) & \stackrel{\alpha_{m+n}}{\rightarrow} & \Omega^{m+n} \Sigma^{m+n}(X \wedge Y)
\end{array}
$$

It is convenient to allow 0 -fold loop spaces $\Omega^{0} \Sigma^{0} X=X$. Let $\mathcal{P}$ be the trivial coefficient system with $\mathscr{P}_{0}=\{*\}, \mathscr{P}_{1}=\{1\}$, and $\mathscr{P}_{q}$ empty for $q>1$. Clearly $C(\mathscr{P}, X)=X$. By convention or definition, $\mathcal{C}_{0}=\mathscr{P}$ and $\mathcal{C}(Y)=\mathscr{P}$ if $Y$ is a point. With $g_{0}$ and $\alpha_{0}$ both identity maps, Lemmas 2.8 and 2.11 are valid for all $m \geqslant 0$ and $n \geqslant 0$.

The following easily checked lemma leads to the context discussed in the previous section.

LEMMA 2.12. The three products displayed in the previous lemma are all homotopy bilinear maps.

We shall also need the following examples. The product of coefficient systems is defined in the evident spacewise way.

Examples 2.13. Sums $(\mathscr{Q}, \mathscr{B}) \rightarrow C$ and $\left(\mathbb{Q}^{\prime}, \mathscr{B}^{\prime}\right) \rightarrow C^{\prime}$ induce a sum $\left(\mathbb{Q} \times \mathbb{Q}^{\prime}, \mathscr{B} \times\right.$ $\left.\mathscr{B}^{\prime}\right) \rightarrow \subset \times C^{\prime}$ such that the two projections define morphisms of sums. The same statement holds for products.

To relate sums and James maps, it is useful to have a notion of a pairing from two sequences of coefficient systems to a third one.

Definition 2.14. Let $\mathbb{Q}^{q}, \mathscr{B}^{q}$ and $e^{q}, q \geqslant 0$, be three sequences of coefficient systems. A pairing $\square:\left(\left\{\mathscr{Q}^{p}\right\},\left\{\mathscr{B}^{q}\right\}\right) \rightarrow\left\{\mathcal{C}^{r}\right\}$ is a collection of maps

$$
\square: \underset{p+q=r}{\chi} \mathbb{Q}_{s_{p}}^{p} \times \mathscr{B}_{t_{q}}^{q} \rightarrow \mathcal{C}_{z}^{r}, \quad z=\sum_{p+q=r} s_{p} t_{q},
$$

one map for each $r \geqslant 0$ and each choice of the $s_{p} \geqslant 0$ and $t_{q} \geqslant 0$, such that the following diagram commutes for morphisms $\phi_{p}: \mathbf{s}_{p} \rightarrow \mathbf{u}_{p}$ and $\psi_{q}: \mathbf{t}_{q} \rightarrow \mathbf{v}_{q}$ in $\Lambda$, $w=\sum_{p+q=r} u_{p} v_{q}:$

$$
\begin{array}{rll}
\underset{p+q=r}{X \mathbb{Q}_{s_{p}}^{p} \times \mathscr{B}_{t_{q}}^{q}} & \stackrel{\square}{\rightarrow} \bigodot_{z} \\
\chi_{p+q=r} \phi_{p} \times \psi_{q} \downarrow & & \downarrow \bigvee_{p+q=r} \phi_{p} \wedge \psi_{q} \\
\underset{p+q=r}{X} \mathbb{Q}_{u_{p}} \times \mathscr{B}_{v_{q}} & \stackrel{\square}{\rightarrow} & \bigodot_{w}
\end{array}
$$


An easy verification gives the following result.

LEMMA 2.15. Let $\square$ be a pairing as above. Let $X_{q}$ and $Y_{q}$ be based spaces, $q \geqslant 0$, and define $W_{r}=\bigvee_{p+q=r} X_{p} \wedge Y_{q}$. There are natural induced maps

$$
\square: \underset{p+q=r}{\chi} A^{p} X_{p} \times B^{q} Y_{q} \rightarrow C^{r} W_{r}
$$

specified on elements by

$$
\underset{p+q=r}{\chi}\left(\left[a_{p} ; x_{p}\right],\left[b_{q} ; y_{q}\right]\right) \rightarrow\left[\square\left(\underset{p+q=r}{X}\left(a_{p}, b_{q}\right)\right) ; \underset{p+q=r(i, j)}{\chi} \underset{\left.\left(x_{p, i} \wedge y_{q, j}\right)\right],}{X}\right.
$$

where $x_{p}=\left(x_{p, 1}, \ldots, x_{p, s_{p}}\right)$ and $y_{q}=\left(y_{q, 1}, \ldots, y_{q, t_{q}}\right)$. The ordering on the right is by increasing $p$ and, for fixed $p$, by lexicographic ordering of the $(i, j)$.

As in the previous section, it is useful to regard these maps $\square$ as components of a single map

$$
\square:\left(\underset{p \geqslant 0}{\chi} A^{p} X_{p}\right) \times\left(\underset{q \geqslant 0}{\chi} B^{q} Y_{q}\right) \rightarrow \underset{r \geqslant 0}{\chi} C^{r} W_{r}
$$

Often we will have canonical maps $f_{r}: W_{r} \rightarrow Z_{r}$, the choice of which should be clear from context. We then continue to write $\square$ for the products obtained by composing with the maps $C^{r} f_{r}$. For example, given a sum $\bigoplus:(Q, \Re) \rightarrow C$ and using the wedge sum of the pairings $D_{p}(Q, X) \wedge D_{q}(\mathscr{B}, X) \rightarrow D_{r}(\mathcal{C}, X)$ of Lemma 2.3 to specify the $f_{r}$, we obtain

$$
\square:\left(\underset{p \geqslant 0}{\chi} A^{p} D_{p}(\mathscr{Q}, X)\right) \times\left(\underset{q \geqslant 0}{\chi} B^{q} D_{q}(\mathscr{B}, X)\right) \rightarrow \underset{r \geqslant 0}{X} C^{r} D_{r}(\mathcal{C}, X) .
$$

In fact, these are the central examples in our applications.

While these last definitions may look a bit formidable, we have already encountered a simple source for such pairings $\square$.

EXAMPLES 2.16. Given further coefficient systems $Q^{p, q}$ and given products $\otimes$ : $\left(\mathbb{Q}^{p}, \mathscr{B}^{q}\right) \rightarrow \mathscr{Q}^{p, q}$ and sums $\bigoplus: X_{p+q=r} \mathscr{Q}^{p, q} \rightarrow \mathrm{C}^{r}$, the following composites define a pairing $\square$ :

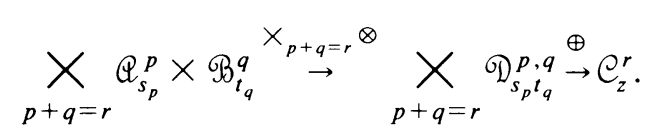

Here the map $\square$ of the previous lemma factors as the corresponding composite. In particular, with $\mathbb{Q}^{r}=\mathscr{B}^{r}=\mathrm{e}^{r}=\mathscr{Q}^{p, q}, p+q=r, \otimes$ and $\bigoplus$ induce pairings $\square$ on the sequences $\left\{C\left(R^{n q}\right)\right\}$ and $\left\{\bigodot_{n q}\right\}$ for any fixed $n$.

Only pairings of this general form are needed for the theorems stated in the introduction. A slight variant not quite of this form will play a role in the general theory.

3. Pairings of James systems and homological splittings. Here we use the pairings to work out how sums and James maps interact. To be precise about this, we must first recall the definition of the latter from [6]. Fix $q \geqslant 0$ and consider an 
injection $\phi: \mathbf{r} \rightarrow \mathbf{s}$. If $\psi: \mathbf{q} \rightarrow \mathbf{r}$ is an ordered injection, so that $\psi(i)<\psi(j)$ if $i<j$, then there is a unique ordered injection $\omega: \mathbf{q} \rightarrow \mathbf{s}$ with the same image as $\phi \circ \psi$. Giving the sets of ordered injections $\mathbf{q} \rightarrow \mathbf{r}$ and $\mathbf{q} \rightarrow \mathbf{s}$, the reverse lexicographic ordering $\left(\psi<\psi^{\prime}\right.$ if $\psi(i)<\psi^{\prime}(i)$ for the largest $i$ with $\left.\psi(i) \neq \psi^{\prime}(i)\right)$, and observing that these sets have $m=(r-q, q)$ and $n=(s-q, q)$ elements, respectively, we see that the correspondence $\psi \rightarrow \omega$ specifies an injection $\bar{\phi}: \mathbf{m} \rightarrow \mathbf{n}$. A James system $\circlearrowright \rightarrow \bigodot^{\prime}$ is a sequence of maps $\xi_{q, r}: \bigodot_{r} \rightarrow \bigodot^{\prime}{ }_{m}, r \geqslant 0$, such that $\xi_{q, r} \phi=\bar{\phi} \xi_{q, s}$ for an injection $\phi: \mathbf{r} \rightarrow \mathbf{s}$. Given such a system, the associated James map $j_{q}: C X \rightarrow$ $C^{\prime} D_{q}(\bigodot, X)$ is specified by the formula

$$
j_{q}[c ; x]=\left[\xi_{q, r}(c) ; \underset{1 \leqslant i \leqslant m}{\chi}\left[c \psi_{i}, \psi_{i}^{*} x\right]\right],
$$

where $c \in \mathcal{C}_{r}, x=\left[x_{1}, \ldots, x_{r}\right] \in X^{r},\left\{\psi_{i}\right\}$ is the set of ordered injections $\mathbf{q} \rightarrow \mathbf{r}$, and $\psi_{i}^{*} x=\left(x_{\psi_{i}(1)}, \ldots, x_{\psi_{i}(q)}\right) \in X^{q}$. When $q=0$, we shall always take $\mathcal{C}^{\prime}=\wp$. Here the definition gives that $j_{0}: C X \rightarrow D_{0}(\circlearrowright, X)=S^{0}$ is constant at 1 . We need some combinatorics to relate these maps to sums.

Combinatorics 3.1. Fix nonnegative integers $r, u$ and $v$, and consider all pairs $(p, q)$ such that $p+q=r$. Let $\left\{\chi_{i}^{p}\right\},\left\{\psi_{j}^{q}\right\}$ and $\left\{\omega_{k}\right\}$ be the ordered sets of ordered injections $\mathbf{p} \rightarrow \mathbf{u}, \mathbf{q} \rightarrow \mathbf{v}$ and $\mathbf{r} \rightarrow \mathbf{u}+\mathbf{v}$, respectively. (Some of these may be empty.) Obviously $\chi_{i}^{p} \vee \psi_{j}^{q} \in\left\{\omega_{k}\right\}$, and it is not much harder to see that any $\omega_{k}$ is $\chi_{i}^{p} \vee \psi_{j}^{q}$ for some choice of $p, q, i$ and $j$. Thus, as unordered sets,

$$
\left\{\omega_{k}\right\}=\coprod_{p+q=r}\left\{\chi_{i}^{p} \vee \psi_{j}^{q}\right\},
$$

this set having $w=(u+v-r, r)=\sum_{p+q=r}(u-p, p)(v-q, q)$ elements. Order $\mathrm{II}_{p+q=r}\left\{\chi_{i}^{p} \vee \psi_{j}^{q}\right\}$ by increasing $p$, for fixed $p$ increasing $i$, and for fixed $p$ and $i$ increasing $j$. Let $\tau_{r, u, v} \in \Sigma_{w}$ be that permutation which reorders this set according to its identification with the ordered set $\left\{\omega_{k}\right\}$.

The following observation may help explain these permutations.

REMARK 3.2. Consider the James construction $M X$ and the classical James maps $j_{q}: M X \rightarrow M X^{[q]}$ (see [9 or 6, 3.3]). With the evident $\oplus$ and $\otimes$, the permutation $\tau_{r, u, v}$ measures the deviation from commutativity of the restriction of the following diagram to $X^{u} \times X^{v}$ :

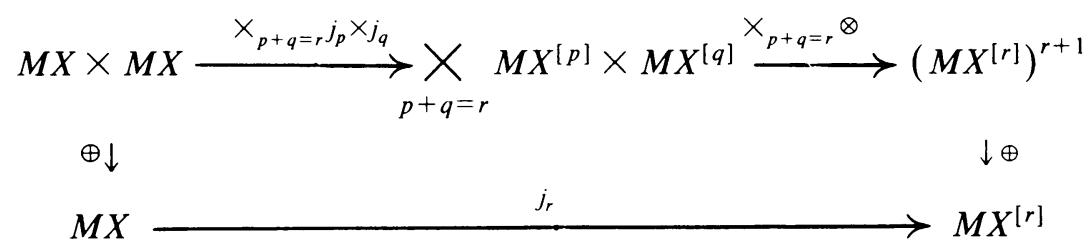

We give a legislative solution to such noncommutativity.

DEFinition 3.3. A pairing of James systems consists of a sum $\oplus:(\mathcal{Q}, \Re) \rightarrow \mathcal{C}$, a pairing $\square:\left(\left\{\mathbb{Q}^{p}\right\},\left\{\mathscr{B}^{q}\right\}\right) \rightarrow\left\{\mathcal{C}^{r}\right\}$, and James systems $\left\{\mu_{p, u}\right\}: \mathbb{Q} \rightarrow \mathbb{Q}^{p},\left\{\nu_{q, v}\right\}:$ $\mathscr{B} \rightarrow \mathscr{B}^{q}$ and $\left\{\xi_{r, w}\right\}: \bigodot \rightarrow e^{r}$, for $p, q, r \geqslant 0$, such that the following diagram commutes for each $r, u$ and $v$, where $w=(u+v-r, r)$ : 


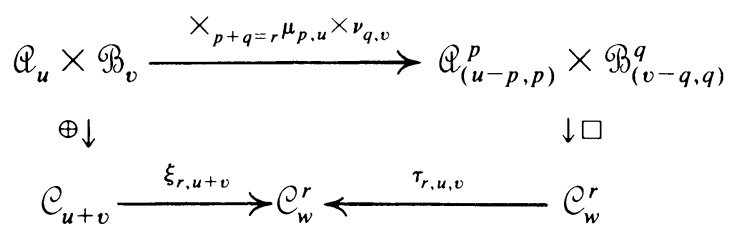

The following result should come as no surprise. What should be mildly surprising is that there are examples.

Proposition 3.4. Given a pairing of James systems, the following diagram commutes for each $r \geqslant 0$ :

$$
\begin{aligned}
& A X \times B Y \stackrel{\times_{p+q=r} j_{p} \times j_{q}}{\longrightarrow} \underset{p+q=r}{X} A^{p} D_{p}(Q, X) \times B^{q} D_{q}(\Re, Y) \\
& \oplus \downarrow \\
& C^{r}\left(\underset{p+q=r}{\vee} D_{p}(\Theta, X) \wedge D_{q}(\mathscr{B}, Y)\right) \\
& \downarrow C^{r}(\oplus) \\
& C(X \vee Y) \longrightarrow j_{r} \longrightarrow C^{r} D_{r}(\circlearrowright, X \vee Y)
\end{aligned}
$$

Proof. It suffices to compute both composites on points $([a ; x],[b ; y])$, where $a \in \mathbb{Q}_{u}, b \in \mathscr{B}_{v}, x \in X^{u}$ and $y \in Y^{v}$. Going clockwise, we obtain

$$
\left[\square\left(\underset{p+q=r}{\chi}\left(\mu_{p, u}(a), v_{q, v}(b)\right)\right) ; \underset{p+q=r(i, j)}{\chi} \underset{X}{X}\left[a \chi_{i}^{p} \oplus b \psi_{j}^{q} ;\left(\chi_{i}^{p}\right)^{*}(x),\left(\psi_{j}^{q}\right)^{*}(y)\right]\right] .
$$

Going counterclockwise, we obtain

$$
\left[\xi_{r, u+v}(a \oplus b) ; \underset{k}{X}\left[(a \oplus b) \omega_{k} ; \omega_{k}^{*}(x, y)\right]\right] .
$$

Since $(a \oplus b)(\chi \vee \psi)=a \chi \vee b \psi$ by the naturality of $\oplus$ and since $(\chi \vee \psi)^{*}(x, y)$ $=\left(\chi^{*}(x), \psi^{*}(y)\right)$ by inspection, the equivariance relation in the construction of $C^{r} D_{r}(\Theta, X \vee Y)$ implies the conclusion.

Our first example is rather trivial, but paradigmatic.

EXAmple 3.5. Let $\oplus:(\mathscr{Q}, \mathscr{B}) \rightarrow \mathcal{C}$ be any sum. As always, take $\mathbb{Q}^{0}=\mathscr{B}^{0}=\mathcal{C}^{0}=\mathscr{P}$. For $q \geqslant 1$, take $\mathbb{Q}^{q}=\mathfrak{B}^{q}=e^{q}=\Re$, where $\Re$ is the operad with each $\Re_{j}$ a single point. The associated construction $N X$ is the infinite symmetric product. Using the only possible maps, we obtain a pairing of James systems. The pairing $\square$ on $\{\Re\}$ is obtained as in Example 2.16 from the only possible sum $\oplus$ and product $\otimes$ on $\Re$.

The rest of this section is an illustrative digression in which we use this example to prove the following analog of Theorem $\mathrm{H}$. 
Theorem 3.6. Let $\oplus:(Q, \Re) \rightarrow \mathcal{C}$ be any sum. For any based spaces $X$ and $Y$ and Abelian group $G$, the following is a natural commutative diagram in which the horizontal arrows are isomorphisms:

$$
\begin{gathered}
\tilde{H}_{*}(A X \times B Y ; G) \stackrel{\Sigma_{r>1} \Sigma_{p+q=r}\left(j_{p} \wedge_{q}\right)_{*}}{\longrightarrow} \sum_{r \geqslant 1} \sum_{p+q=r} \tilde{H}_{*}\left(D_{p}(\mathcal{Q}, X) \wedge D_{q}(\mathscr{B}, Y) ; G\right) \\
\tilde{H}_{*}(C(X \vee Y) ; G) \stackrel{\Sigma_{r>1}\left(j_{r}\right)_{*}}{\longrightarrow} \underset{r \geqslant 1}{\longrightarrow} \tilde{H}_{*}\left(D_{r}(\mathcal{C}, X \vee Y) ; G\right)
\end{gathered}
$$

Proof. For $r \geqslant 1$, the present special case of the diagram in Proposition 3.4 can be rewritten in the form:

$$
\begin{aligned}
& A X \times B Y \stackrel{\Sigma_{p+q=r} j_{p} \times j_{q}}{\longrightarrow} N\left(\underset{p+q=r}{\vee} D_{p}(\mathscr{Q}, X) \wedge D_{q}(\Re, Y)\right) \\
& \oplus \downarrow \quad \downarrow N(\oplus) \\
& C(X \vee Y) \longrightarrow N D_{r}(\bigodot, X \vee Y)
\end{aligned}
$$

Let $M G$ denote the Moore space with $H_{1}(M G ; Z)=G$, so that $\tilde{H}_{q}(X ; G)$ is isomorphic to $\pi_{q+1} N(M G \wedge X)$ for any based space $X$. The inclusion of $M G$ in $N(M G)$ and the tensor product $N(M G) \wedge N X \rightarrow N(M G \wedge X)$ yield a natural map $M G \wedge N X \rightarrow N(M G \wedge X)$ and the addition on $N X$ yields a natural map $N N X \rightarrow N X$. Smashing our diagram with $M G$, applying $N$, and using these maps on the right, we obtain the diagram:

$$
\begin{array}{ccc}
N(M G \wedge(A X \times B Y)) & \rightarrow & N\left(\underset{p+q=r}{\vee} M G \wedge D_{p}(\mathscr{Q}, X) \wedge D_{q}(\mathscr{B}, Y)\right) \\
\downarrow & & \downarrow \\
N(M G \wedge C(X \vee Y)) & \rightarrow & N\left(M G \wedge D_{r}(\mathcal{C}, X \vee Y)\right)
\end{array}
$$

Summing over $r \geqslant 1$, we can replace the spaces to which $N$ is applied on the right by the wedges over $r \geqslant 1$ of all these spaces. It was proven in [6, §4] that the resulting bottom arrow induces an isomorphism on homotopy groups, and a precisely analogous induction over filtration gives the same conclusion for the resulting top arrow.

The interest lies in the following immediate consequence.

COROLlaRY 3.7. For any commutative coefficient ring, the pairing

$$
\oplus_{*}: \tilde{H}_{*} A X \otimes \tilde{H}_{*} B X \rightarrow \tilde{H}_{*} C X
$$

can be computed as the sum of the filtration quotient pairings

$$
\bigoplus_{*}: \tilde{H}_{*} D_{p}(\Theta, X) \otimes \tilde{H}_{*} D_{q}(\Re, X) \rightarrow \tilde{H}_{*} D_{p+q}(\bigodot, X) \text {. }
$$

Note, in particular, the case $\oplus:(\Re, \Re) \rightarrow \Re$. Here $D_{p}(\Re, X)$ is the reduced symmetric power $X^{[p]} / \Sigma_{p}$. 
4. Canonical pairings and splittings of spectra. Here we obtain canonical pairings of James systems and use them to prove a generalization of Theorem $\mathrm{H}$.

Any coefficient system $\bigodot$ has an associated James system $j_{q}: \bigodot \rightarrow \mathscr{P}\left(\bar{\bigodot}_{q}\right)$, where $\bar{\complement}_{q}=\bigodot_{q} / \Sigma_{q}$ (denoted $\Re_{q}$ in [6]) and where $\mathscr{P}(Y)$ denotes the coefficient system whose $j$ th space is the Cartesian power $Y^{j}$. Indeed, let $\pi: \bigodot_{q} \rightarrow \bar{\bigodot}_{q}$ be the projection on orbits and define $\xi_{q, r}: \bigodot_{r} \rightarrow \bar{\complement}_{q}^{m}, m=(r-q, q)$, by

$$
\xi_{q, r}(c)=\left(\pi\left(c \psi_{1}\right), \ldots, \pi\left(c \psi_{m}\right)\right)
$$

where $\left\{\psi_{i}\right\}$ is the ordered set of ordered injections $\mathbf{q} \rightarrow \mathbf{r}$. We would rather land in the configuration space coefficient system $\mathcal{C}\left(\bar{\complement}_{q}\right)$. Thus we say that $\mathcal{C}$ is separated if $\xi_{q, r}$ takes values in $F\left(\bar{\bigodot}_{q}, m\right)$ for all $q$ and $r$. We then refer to $\left\{\xi_{q, r}\right\}: \circlearrowright \rightarrow C\left(\bar{\complement}_{q}\right)$ as a canonical James system. It induces a canonical James map

$$
j_{q}: C X \rightarrow C\left(\bar{\bigodot}_{q}, D_{q}(\bigodot, X)\right) .
$$

For examples, it is easily checked that $e_{n}$ is separated if $n \geqslant 1$ and $C(Y)$ is separated if $Y$ has infinitely many points.

Now let $\oplus:(\mathscr{Q}, \mathscr{B}) \rightarrow C$ be a sum and let $\mathscr{Q}^{p, q}=\mathcal{C}\left(\bar{Q}_{p} \times \overline{\mathscr{B}}_{q}\right)$. By Example 2.9, we have a product $\otimes:\left(e\left(\overline{\mathscr{Q}}_{p}\right), e\left(\overline{\mathscr{G}}_{q}\right)\right) \rightarrow \mathscr{Q}^{p, q}$. As in Example 2.5, the maps $\overline{\mathbb{Q}}_{p} \times \overline{\mathscr{G}}_{q} \rightarrow \overline{\mathcal{C}}_{p+q}$ induced by $\oplus$ give rise to an $(r+1)$-fold sum $\boxplus: X_{p+q=r} \mathscr{Q}^{p, q} \rightarrow$ $\mathcal{P}\left(\overline{\mathcal{C}}_{r}\right)$. By Example 2.16, there results a pairing

$$
\square:\left(\left\{\Theta\left(\overline{\mathscr{Q}}_{p}\right)\right\},\left\{\Theta\left(\overline{\mathscr{B}}_{q}\right)\right\}\right) \rightarrow\left\{\mathscr{P}\left(\overline{\mathcal{C}}_{r}\right)\right\} .
$$

Again, we would rather land in $\left\{\mathcal{C}\left(\bar{\complement}_{r}\right)\right\}$.

Definition 4.1. Let $Q, \mathscr{B}$ and $C$ be separated coefficient systems. A sum $\oplus$ : $(Q, \mathscr{B}) \rightarrow \mathcal{C}$ is said to be separated if the induced maps

$$
\oplus: \coprod_{p+q=r} \bar{Q}_{p} \times \bar{\Re}_{q} \rightarrow \bar{\complement}_{r}
$$

are injections and so induce a sum $\oplus: X_{p+q=r} \mathscr{D}^{p, q} \rightarrow \mathcal{C}\left(\bar{\complement}_{r}\right)$. A sum is said to be weakly separated if the pairing $\square$, defined above, factors through $\left\{\varrho\left(\bar{\bigodot}_{r}\right)\right\}$. We then refer to

$$
\square:\left(\left\{e\left(\overline{\mathscr{Q}}_{p}\right)\right\},\left\{\Theta\left(\overline{\mathscr{B}}_{q}\right)\right\}\right) \rightarrow\left\{e\left(\bar{C}_{r}\right)\right\}
$$

as a canonical pairing of James systems.

We pause to check that we have gotten everything right.

Proposition 4.2. If $\oplus:(\mathscr{Q}, \mathscr{B}) \rightarrow \bigodot$ is a weakly separated sum, then the canonical James systems and the canonical pairing constitute a pairing of James systems.

Proof. Consider the diagram of Definition 3.3. For $a \in \mathbb{Q}_{u}$ and $b \in \mathscr{B}_{v}$,

$$
\square\left(\underset{p+q=r}{\chi}\left(\mu_{p, u}(a), \nu_{q, v}(b)\right)\right)=\left\langle\underset{p+q=r(i, j)}{X} \underset{\left.X\left(a \chi_{i}^{p}\right) \oplus \pi\left(b \psi_{j}^{q}\right)\right\rangle}{\chi}\right.
$$

while

$$
\xi_{r, u+v}(a+b)=\left\langle\chi_{k} \pi\left((a \oplus b) \omega_{k}\right)\right\rangle .
$$

The permutation $\tau_{r, u, v}$ was defined so as to convert the first to the second. 
ExAmple 4.3. The sum $\oplus:(\mathcal{C}(Y), \mathcal{C}(Z)) \rightarrow \mathcal{C}(Y \amalg Z)$ of Example 2.5 is separated for (infinite) spaces $Y$ and $Z$. Indeed, the induced maps

$$
\oplus: \coprod_{p+q=r} B(Y, p) \times B(Z, q) \rightarrow B(Y \amalg Z, r)
$$

are easily seen to be homeomorphisms.

EXAMPLE 4.4. The operad sum on $\bigodot_{n}$ is separated for $n \geqslant 1$.

EXAMPLE 4.5. If either of two sums $(Q, \mathscr{B}) \rightarrow C$ and $\left(\mathbb{Q}^{\prime}, \mathscr{B}^{\prime}\right) \rightarrow C^{\prime}$ is weakly separated, then so is the product sum $\left(\mathbb{Q} \times \mathbb{Q}^{\prime}, \mathfrak{B} \times \mathfrak{B}^{\prime}\right) \rightarrow \mathcal{C} \times \mathcal{C}^{\prime}$. The analogous assertion for separated sums is false, and this motivates our introduction of the weaker notion.

In the rest of this section, we use this example to prove the following result. With $Q=\mathscr{B}=\mathcal{C}=\mathcal{C}\left(R^{n}\right)$ and $X=Y$, we see from Lemma 2.8 that Theorem $\mathrm{H}$ is an immediate consequence. A coefficient system $C$ is said to be $\Sigma$-free if each $C_{j}$ is $\Sigma_{j}$-free.

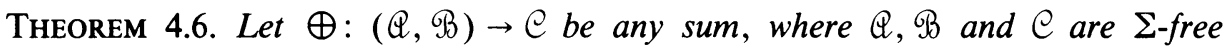
coefficient systems. For based spaces $X$ and $Y$, the following is a natural commutative diagram in the stable category in which the horizontal arrows are equivalences:

$$
\begin{aligned}
& \Sigma^{\infty}(A X \times B Y) \stackrel{\Sigma_{r>1} \Sigma_{p+q=r} j_{p} \wedge j_{q}}{\longrightarrow} \underset{r \geqslant 1}{\vee} \underset{p+q=r}{\vee} \Sigma^{\infty}\left(D_{p}(Q, X) \wedge D_{p}(\Re, Y)\right) \\
& \Sigma^{\infty} \oplus \downarrow \quad \downarrow \vee_{r \geqslant 1} \vee_{p+q=r} \oplus \\
& \Sigma^{\infty} C(X \vee Y) \longrightarrow \Sigma_{r>1} j_{r} \longrightarrow \underset{r \geq 1}{\vee} \Sigma^{\infty} D_{r}(\mathcal{C}, X \vee Y)
\end{aligned}
$$

Proof. Let $\tilde{e}=\bigodot \times \bigodot_{\infty}$. (We used $e\left(R^{\infty}\right)$ rather than $\bigodot_{\infty}$ for the same idea in [6]; use of $C_{\infty}$ is slightly more efficient.) By [6,2.7], the projections $\tilde{C} X \rightarrow C X$ and $D_{q}(\tilde{e}, X) \rightarrow D_{q}(\mathcal{C}, X)$ are equivalences, and similarly for $\mathscr{Q}$ and $\mathscr{B}$. The separated $\operatorname{sum}\left(\bigodot_{\infty}, e_{\infty}\right) \rightarrow e_{\infty}$ and the given sum induce a weakly separated $\operatorname{sum}(\tilde{\mathscr{Q}}, \tilde{\mathscr{G}}) \rightarrow \tilde{\mathcal{C}}$. To avoid drowning in a wave of tildes, we start over and assume, without loss of generality, that the given sum is weakly separated. Then Proposition 3.4 applies. To get from its diagram to a diagram in the stable category, we apply the tilde construction to the coefficient systems $\mathcal{C}\left(\bar{\complement}_{q}\right)$ to obtain natural maps

$$
C\left(\overline{\mathcal{C}}_{r}, X\right) \stackrel{\pi_{1}}{\leftarrow} C\left(\tilde{\mathcal{C}}\left(\overline{\mathcal{C}}_{r}\right), X\right) \stackrel{\pi_{2}}{\rightarrow} C_{\infty} X \stackrel{\alpha_{\infty}}{\rightarrow} Q X
$$

Again, the projection $\pi_{1}$ is an equivalence. Choose a homotopy inverse for each $X$ and let $\beta: C\left(\overline{\mathcal{C}}_{r}, X\right) \rightarrow Q X$ be the resulting map. Observe that the three sequences of projections

$$
\mathrm{e}\left(\overline{\mathscr{Q}}_{p}\right) \leftarrow \tilde{e}\left(\overline{\mathscr{Q}}_{p}\right) \rightarrow \mathcal{C}_{\infty}, \quad e\left(\overline{\mathscr{B}}_{q}\right) \leftarrow \tilde{e}\left(\overline{\mathscr{S}}_{q}\right) \rightarrow \mathcal{C}_{\infty}, \quad e\left(\bar{e}_{q}\right) \leftarrow \tilde{e}(\overline{\mathcal{C}}) \rightarrow e_{\infty}
$$

specify two morphisms of pairings $\square$. Composing the diagram of Proposition 3.4 with maps $\beta$ on the right, we find by Lemmas 2.8 and 2.11 and the observation just 
given that the resulting homotopy commutative diagram may be rewritten in the form

$$
\begin{gathered}
A X \times B Y \stackrel{\times_{p+q=r} j_{p} \wedge j_{q}}{\longrightarrow} \times \underset{p+q=r}{\longrightarrow} Q D_{p}(Q, X) \wedge Q D_{q}(\mathscr{G}, Y) \stackrel{\square}{\longrightarrow} Q\left(\underset{p+q=r}{\vee} D_{p}(Q, X) \wedge D_{q}(\mathscr{G}, Y)\right) \\
\quad \begin{array}{l}
\downarrow \downarrow \\
C(X \vee Y) \longrightarrow
\end{array} \\
j_{r} Q D_{r}(\Theta, X \vee Y)
\end{gathered}
$$

where we have continued to write $j_{r}$ for its composite with $\beta$ and where $\square$ denotes the composite obtained from external smash products and the $(r+1)$-fold loop sum. Adjointing to obtain maps of suspension spectra, commuting wedges past $\Sigma^{\infty}$, and summing over $r \geqslant 1$, we obtain the diagram of the statement. Its bottom arrow was proven to be an equivalence of spectra in $[6, \S 8]$, and a precisely analogous induction over filtration proves the same conclusion for the top arrow. Naturality in $X$ and $Y$ is clear and naturality in the given pairing is proven by the methods of $[6, \S 9]$.

5. Multiplicative properties of the unstable James maps. We prove Theorems A and $\mathrm{G}$ here. To begin, specialize the theory of the previous section to the canonical pairing of James systems associated to the composite of the separated sum $\left(\mathcal{C}\left(R^{n}\right), \mathcal{C}\left(R^{n}\right)\right) \rightarrow \mathcal{C}\left(R^{n} \amalg R^{n}\right)$ and $\mathcal{C}(i)$ for a chosen good embedding $i=i_{1}+i_{2}$ : $R^{n} \amalg R^{n} \rightarrow R^{n}$. To abbreviate notation, set

$$
e(n, q)=e\left(B\left(R^{n}, q\right)\right) \text { and } C(n, q, X)=C\left(B\left(R^{n}, q\right), D_{q}\left(R^{n}, X\right)\right) .
$$

Notice that $C(n, q, X)$ is an $H$-space by virtue of the good embedding

$$
e(i)=B\left(i_{1}, q\right)+B\left(i_{2}, q\right): B\left(R^{n}, q\right) \amalg B\left(R^{n}, q\right) \rightarrow B\left(R^{n}, q\right)
$$

induced by $i$. Proposition 3.4 specializes to give the following result.

Proposition 5.1. The following diagram is commutative:

$$
\begin{gathered}
C\left(R^{n}, X\right) \times C\left(R^{n}, X\right) \stackrel{\times_{p+q=r} j_{p} \times j_{q}}{\longrightarrow} \underset{p+q=r}{X} C(n, p, X) \times C(n, q, X) \\
\qquad \downarrow \\
C\left(R^{n}, X\right) \longrightarrow \downarrow^{-}
\end{gathered}
$$

The maps $\square$ arise from the pairing on the sequence of coefficient systems $\mathcal{C}(n, q)$ obtained by specialization of Definition 4.1. It is helpful to notice that there is a slightly different pairing which gives rise to homotopic maps. We have the internalized tensor product pairings

$$
(\bigodot(n, p), \mathcal{C}(n, q)) \stackrel{\otimes}{\rightarrow} \mathcal{C}\left(B\left(R^{n}, p\right) \times B\left(R^{n}, q\right)\right) \stackrel{\varrho(\oplus)}{\rightarrow} \bigodot(n, p+q)
$$

and, as above, a good embedding $j: \amalg_{p+q=r} R^{n} \rightarrow R^{n}$ induces a good embedding $e(j): \amalg_{p+q=r} B\left(R^{n}, r\right) \rightarrow B\left(R^{n}, r\right)$ and thus an $(r+1)$-fold sum $\bigoplus: C(n, r)^{r+1} \rightarrow$ $\mathcal{C}(n, r)$. Therefore, application of Example 2.16 gives a second pairing on $\{C(n, q)\}$. The following observation leads to a comparison. 
LEMMA 5.2. If $n \geqslant 2$, the following composites are isotopic:

$$
\coprod_{p+q=r} B\left(R^{n}, p\right) \times B\left(R^{n}, q\right) \stackrel{\amalg_{p+q \doteq r}^{\oplus}}{\rightarrow} \coprod_{p+q=r} B\left(R^{n}, r\right) \underset{e(j)}{\stackrel{\nabla}{\Rightarrow}} B\left(R^{n}, r\right) .
$$

Proof. By Lemma 2.6, we may as well choose our good embeddings $i=i_{1}+i_{2}$ and $j=j_{0}+\cdots+j_{r}$ so that there exist isotopies $J_{p}: j_{p} \simeq 1$ such that each embedding $J_{p, t}, 0 \leqslant t \leqslant 1$, carries the images of $i_{1}$ and $i_{2}$ into themselves. With these choices, the conclusion is obvious since the respective composites send $(a, b)$ to $\left(i_{1} a, i_{2} b\right)$ and $\left(j_{p} i_{1} a, j_{p} i_{2} b\right)$ if $a \in B\left(R^{n}, p\right)$ and $b \in B\left(R^{n}, q\right)$, the cited embeddings $R^{n} \rightarrow R^{n}$ being applied coordinatewise.

A simple comparison of definitions gives the following consequence.

LEMma 5.3. The map $\square: X_{p+q=r} C(n, p, X) \times C(n, q, X) \rightarrow C(n, r, X)$ is homotopic to the composite

$$
\underset{p+q=r}{X} C(n, p, X) \times C(n, q, X) \stackrel{\times_{p+q=r} \otimes}{\longrightarrow} \underset{p+q=r}{X} C(n, r, X) \stackrel{\oplus}{\rightarrow} C(n, r, X) .
$$

It is easy to see that the maps $\otimes$ here are homotopy bilinear, and this implies the first part of the following result. Choose embeddings $e_{q}: B\left(R^{n}, q\right) \rightarrow R^{2 n q}$; there exist such embeddings since $B\left(R^{n}, q\right)$ is an $n q$-manifold, and any two are isotopic.

Proposition 5.4. For $n \geqslant 2, X_{q \geqslant 0} C(n, q, X)$ is an H-semiring space and the product over $q \geqslant 0$ of the maps

$$
\beta_{2 n q} C\left(e_{q}, 1\right): C(n, q, X) \rightarrow \Omega^{2 n q} \Sigma^{2 n q} D_{q}\left(R^{n}, X\right)
$$

is a map of $H$-semiring spaces.

Proof. Recall that $\beta_{n}=\alpha_{n} g_{n}^{-1}$. In view of Lemmas 2.8 and 2.11 , it suffices to study the maps $C\left(e_{q}, 1\right)$. They are $H$-maps since the $H$-space structure on $C\left(R^{2 n q}, D_{q}\left(R^{n}, X\right)\right)$ can be obtained from a good embedding $j=j_{1}+j_{2}$ : $R^{2 n q} \amalg R^{2 n q} \rightarrow R^{2 n q}$ such that $j_{k} e_{q} \simeq e_{q} B\left(i_{k}, q\right), k=1$ and 2 , via isotopies with disjoint images. Similarly, up to homotopy, $C\left(e_{r}, 1\right)$ carries the $(r+1)$-fold sum on $C(n, r, X)$ to that on $C\left(R^{2 n r}, D_{r}\left(R^{n}, X\right)\right)$. If $p+q=r, e_{r} \circ \oplus$ is isotopic to $e_{p} \times e_{q}: B\left(R^{n}, p\right) \times B\left(R^{n}, q\right) \rightarrow R^{2 n r}$. This and the naturality of the external pairing $\otimes$ imply

$$
\otimes \circ\left(C\left(e_{p}, 1\right) \times C\left(e_{q}, 1\right)\right) \simeq C\left(e_{r}, 1\right) \circ \otimes .
$$

If $X$ is connected, the proof of Theorem A can now be completed as follows. We can choose $e_{q}$ to factor through $e_{q}^{\prime}: B\left(R^{n}, q\right) \rightarrow R^{s}$, where $s=s(n, q)$ is the embedding dimension of $B\left(R^{n}, q\right)$, and we can take the James maps needed for Theorem $\mathrm{A}$ to be the composites

$$
\Omega^{n} \Sigma^{n} X \stackrel{\beta_{n}^{-1}}{\rightarrow} C\left(R^{n}, X\right) \stackrel{j_{q}}{\rightarrow} C(n, q, X) \stackrel{\beta_{s} C\left(e_{q}^{\prime}, 1\right)}{\longrightarrow} \Omega^{s} \Sigma^{s} D_{q}\left(R^{n}, X\right) .
$$

Here part (3) of Theorem A is valid for all CW-complexes, not just finite ones. We observed in [6, 5.7] that $B\left(R^{n}, q\right)$ is diffeomorphic to $R \times A(n, q)$, where

$$
A(n, q)=R^{n-1} \times F\left(R^{n}-\{0\}, q-1\right) / \Sigma_{q} .
$$


Since we defined $t(n, q)$ to be 1 plus the embedding dimension of $A(n, q), s(n, q)$ might be a smidgeon smaller.

Before proceeding, we interpolate a lemma. Via the diffeomorphism just cited, a good embedding $i: R^{n} \amalg R^{n} \rightarrow R^{n}$ determines the good embedding

$$
f(i): B\left(R^{n}, q\right) \amalg B\left(R^{n}, q\right) \rightarrow B\left(R^{n}, q\right)
$$

whose restrictions are $i_{1} \times 1$ and $i_{2} \times 1$. We shall prove the following analog of Lemma 2.6 in the appendix.

LEMMA 5.5. The good embeddings $e(i)$ and $f(i)$ are isotopic.

Thus $f(i)$ induces the $H$-space structure used above on $C(n, q, X)$. We agree to let our good embeddings $i$ (for any $n)$ have the form $\left(h_{1} \times 1\right)+\left(h_{2} \times 1\right)$ for a good embedding $h_{1}+h_{2}: R \amalg R \rightarrow R$. Now choose an embedding $c_{q}: A(n, q) \rightarrow R^{t-1}$, $t=t(n, q)$, and let $d_{q}=1 \times c_{q}$ be the resulting embedding of $B\left(R^{n}, q\right)$ in $R^{t}$. Then $d_{q}$ clearly commutes with the good embeddings $f(i)$ for $B\left(R^{n}, q\right)$ and $i$ for $R^{t}$. Thus $C\left(d_{q}, 1\right)$ is an $H$-map, hence so is the composite

$$
\gamma_{t}=\beta_{t} C\left(d_{q}, 1\right): C(n, q, X) \rightarrow \Omega^{t} \Sigma^{t} D_{q}\left(R^{n}, X\right) .
$$

We now prove Theorem G. Let $p$ be an odd prime. Calculations of Cohen [5, III] give that, with mod $p$ coefficients,

$$
\tilde{H}_{*} D_{q}\left(R^{2 n}, S^{2 k-1}\right)=0 \quad \text { if } 1<q<p \quad \text { and } \quad \tilde{H}_{*} D_{p}\left(R^{2}, S^{2 k-1}\right)=\tilde{H}_{*} P^{2 p k-1}(p) \text {, }
$$

the latter with nontrivial Bockstein on both sides. (If $n>1, D_{p}\left(R^{2 n}, S^{2 k-1}\right)$ has too much homology to be a Moore space.) Thus, $p$-locally,

$$
D_{q}\left(R^{2 n}, S^{2 k-1}\right) \simeq\{*\} \quad \text { if } 1<q<p \quad \text { and } \quad D_{p}\left(R^{2}, S^{2 k-1}\right) \simeq P^{2 p k-1}(p) ;
$$

the first of these implies

$$
D_{q}\left(R^{2 n}, S^{2 k-1}\right) \wedge D_{p-q}\left(R^{2 n}, S^{2 k-1}\right) \simeq\{*\} \quad \text { if } 0<q<p .
$$

By Proposition 5.1, Lemma 5.3, and the observations just given, the composite James map

$$
\Omega^{2 n} S^{2 n+2 k-1} \stackrel{\beta_{2 n}^{-1}}{\rightarrow} C\left(R^{2 n}, S^{2 k-1}\right) \stackrel{j_{p}}{\rightarrow} C\left(2 n, p, S^{2 k-1}\right) \stackrel{\gamma_{t}}{\rightarrow} \Omega^{t} \Sigma^{t} D_{p}\left(R^{2 n}, S^{2 k-1}\right)
$$

becomes an $H$-map when localized at $p$.

To prove Theorem A for nonconnected spaces, we exploit the following generalization, due to Caruso [4], of the results cited in §2. (A space $Y$ is weak metric if $\Delta Y=d^{-1}(0)$ for some map $d: Y \times Y \rightarrow[0, \infty)$.)

THEOREM 5.6. For weak metric spaces $Y$, there is a natural $H$-map $\beta: C(R \times Y, X)$ $\rightarrow \Omega C(Y, \Sigma X)$ which is a homotopy equivalence if $X$ is connected and is a group completion in general, provided that, for the latter, $Y$ has the form $R \times Z$.

With $Y=R^{n-1}$, iteration gives our map $\beta_{n}: C\left(R^{n}, X\right) \rightarrow \Omega^{n} \Sigma^{n} X$; that is, $\beta_{n} \simeq$ $\left(\Omega \beta_{n-1}\right) \beta$. We consider the spaces $Y=A(n, q)$ and we set

$$
G(n, q, X)=\Omega C\left(A(n, q), \Sigma D_{q}\left(R^{n}, X\right)\right)
$$


to abbreviate notation. Thus we have group completions $\beta: C(n, q, X) \rightarrow G(n, q, X)$. By Proposition 5.4, Corollary 1.7 applies to give $X_{q \geqslant 0} G(n, q, X)$ a structure of weak $H$-semiring space such that $\chi_{q \geqslant 0} \beta$ is a map of weak $H$-semiring spaces. Moreover, if we define

$$
\delta_{t}=\beta_{t-1} \circ C\left(c_{q}, 1\right): C\left(A(n, q), \Sigma D_{q}\left(R^{n}, X\right)\right) \rightarrow \Omega^{t-1} \Sigma^{t} D_{q}\left(R^{n}, X\right),
$$

then the following diagram of $H$-maps is homotopy commutative:

$$
\begin{array}{ccc}
C(n, q, X) & \stackrel{\beta}{\rightarrow} & G(n, q, X) \\
\gamma_{t} \searrow & & \swarrow \Omega \delta_{t} \\
& \Omega^{t} \Sigma^{t} D_{q}\left(R^{n}, X\right) &
\end{array}
$$

After composition with $\eta[t, 2 n q]$, this $H$-map agrees with the $H$-map $\beta_{2 n q} C\left(e_{q}, 1\right)$ of Proposition 5.4. It follows by the uniqueness clause of Proposition 1.4 that the product over $q \geqslant 0$ of the maps $\eta[t, 2 n q] \Omega \delta_{t}$ is also a map of weak $H$-semiring spaces.

By Corollary 1.7 again, the unit $H$-space $\{1\} \times\left(X_{q \geqslant 1} G(n, q, X)\right)$ is grouplike. By Proposition 5.1, the composites $\beta j_{q}$ are the components of an $H$-map from $C\left(R^{n}, X\right)$ to this unit space. By Proposition 1.2, this $H$-map extends over the group completion $\Omega^{n} \Sigma^{n} X$. When composed with the maps $\Omega \delta_{t}$, the components of the extended weak $H$-map provide the James maps $\Omega^{n} \Sigma^{n} X \rightarrow \Omega^{t} \Sigma^{t} D_{q}\left(R^{n}, X\right)$ promised in Theorem A. Part (1) of that result is obvious, part (2) holds by uniqueness since $j_{1}$ is clearly an additive $H$-map, and part (3) follows from the preceding paragraph.

6. The Segal maps and the partial powers maps. Here we prove (1)-(3) of Theorem B and the combinatorial level of (1)-(3) of Theorem C.

The combinatorics in the rest of the paper are simplified by use of the very pretty way of thinking about the spaces $C(Y, X)$ introduced by Koschorke and Sanderson [11]. They pointed out that $C(Y, X)$ can be described as the set of pairs $\langle A, f\rangle$, where $A$ is a finite subset of $Y$ and $f: A \rightarrow X$ is a function; $\langle A, f\rangle$ and $\left\langle A^{\prime}, f^{\prime}\right\rangle$ are identified if $f=f^{\prime}$ on $A \cap A^{\prime}$ and $f$ and $f^{\prime}$ both carry all points of their domains not in $A \cap A^{\prime}$ to the basepoint of $X$. The same notation will be used for elements of the subquotients $D_{q}(Y, X)$.

With this description, the canonical James map

$$
j_{q}: C(Y, X) \rightarrow C\left(B(Y, q), D_{q}(Y, X)\right)
$$

takes the pleasant form $j_{q}\langle A, f\rangle=\langle B, g\rangle$, where $B \subset B(Y, q)$ is the set of subsets $\alpha$ of $A$ with $q$ elements and $g: B \rightarrow D_{q}(Y, X)$ maps $\alpha$ to the point $\langle\alpha, f \mid \alpha\rangle$. Here we regard $B(Y, q)$ as the set of subsets of $Y$ with $q$ elements; we regard $F(Y, q)$ as the set of ordered subsets of $Y$ with $q$ elements.

We could define Segal maps $D_{q}(\bigodot, X) \rightarrow C\left(\bigodot_{q}, X^{[q]}\right)$ for any $\Sigma$-free coefficient system $\mathcal{C}$, but we restrict to configuration space systems since these provide all the examples we need and allow full exploitation of the framework just established.

Definition 6.1. Define the $q$ th Segal map $s_{q}: D_{q}(Y, X) \rightarrow C\left(F(Y, q), X^{[q]}\right)$ by $s_{q}\langle A, f\rangle=\left\langle F(A, q), f^{[q]} \mid F(A, q)\right\rangle$. Less cryptically, $F(A, q)$ is the set of all 
orderings of the unordered set $A$. By convention, $s_{0}$ is the identity map of $S^{0}$. Clearly, $s_{1}$ is the inclusion of the first filtration and $s_{q}$ takes values in the $(q$ !)th filtration.

The Segal maps $D_{q}\left(R^{n}, X\right) \rightarrow \Omega^{n q} \sum^{n q} X^{[q]}$ of Theorem B are obtained by setting $Y=R^{n}$, including $F\left(R^{n}, q\right)$ in $R^{n q}$, and applying $\beta_{n q}$. Parts (1) and (2) of that result are obvious, and we next prove (3). For $b \in F(Y, q)$, define $\Delta_{b}: X \rightarrow D_{q}(Y, X)$ by $\Delta_{b}(x)=[b ; \Delta(x)]$. Then the composite $s_{q} \circ \Delta_{b}: X \rightarrow C\left(Y^{q}, X^{[q]}\right)$ sends a point $x$ to $\langle A, f\rangle$, where $A=\left\{b \sigma \mid \sigma \in \Sigma_{q}\right\}$ and $f(b \sigma)=\Delta(x)$ for all $\sigma$. When $Y=R^{n}$, we can choose a good embedding $i_{1}+\cdots+i_{q \text { ! }}$ of the disjoint union of $q$ ! copies of $R^{n q}$ in $R^{n q}$ such that $i_{k}(0)=b \sigma_{k}$ for some chosen ordering of the elements $\sigma_{k} \in \Sigma_{q}$. Then $s_{q} \circ \Delta_{b}=q !(\eta \circ \Delta)$, where $\eta: X^{[q]} \rightarrow C\left(R^{n q}, X^{[q]}\right)$ sends $y$ to $[\langle 0\rangle ; y]$. The desired formula follows by use of Lemma 2.8 .

It is not the Segal maps of Theorem B, but their $t$-fold loop extensions, that are of real interest, and we need a combinatorial model for the latter. The following definitions give the relevant maps.

Definitions 6.2. (i) For spaces $Y$ and $Z$, define $\zeta: C(Y, C(Z, X)) \rightarrow C(Z \times Y, X)$ by $\zeta\langle A, f\rangle=\langle C, h\rangle$, where if $f(a)=\left\langle B_{a}, g_{a}\right\rangle$ for $a \in A$, then

$$
C=\bigcup_{a \in A} B_{a} \times\{a\} \subset Z \times Y \text { and } h(b, a)=g_{a}(b) \text { for } b \in B_{a} .
$$

(ii) Define $\zeta: C_{m} C_{n} X \rightarrow C_{m+n} X$ by the formula

$$
\zeta\left[c ; \underset{r=1}{X}\left[d_{r} ; y_{r}\right]\right]=\left[\left\langle\underset{r=1}{X} \underset{s=1}{X} \chi_{k}^{j_{k}}\left(d_{r, s} \times c_{r}\right)\right\rangle ; \underset{r=1}{X} y_{r}\right],
$$

where $c=\left\langle c_{1}, \ldots, c_{k}\right\rangle \in \mathcal{C}_{m, k}, d_{r}=\left\langle d_{r, 1}, \ldots, d_{r, j_{r}}\right\rangle \in \mathcal{C}_{n, j_{r}}, y_{r} \in X^{j_{r}}$, and the $d_{r, s} \times c_{r}$ are product little $(m+n)$-cubes.

(iii) Define $\zeta=\Omega^{m} \nu: \Omega^{m} \Sigma^{m} \Omega^{n} \Sigma^{n} X \rightarrow \Omega^{m+n} \Sigma^{m+n} X$, where $\nu(g \wedge u)(t)=g(t) \wedge u$ for $g \in \Omega^{n} \Sigma^{n} X, u \in S^{m}$ and $t \in S^{n}$. Thus, for $f \in \Omega^{m} \Sigma^{m} \Omega^{n} \Sigma^{n} X$ and $s \in S^{m}$,

$$
\zeta(f)(t \wedge s)=g(t) \wedge u \text { if } f(s)=g \wedge u .
$$

We have written out formulas because more conceptual formulations tend to obscure the orderings of loop coordinates. With these formulas, the following lemma is easily checked.

LEMMA 6.3. The following diagram is commutative:

$$
\begin{gathered}
C\left(R^{m}, C\left(R^{n}, X\right)\right) \stackrel{g_{m} \circ C_{m} g_{n}}{\stackrel{\alpha_{m}}{\longleftarrow}} C_{m} C_{n} X \stackrel{\alpha_{m} \circ C_{m} \alpha_{n}}{\longrightarrow} \Omega^{m} \sum^{m} \Omega^{n} \Sigma^{n} X \\
\downarrow \downarrow \\
\downarrow \zeta \\
C\left(R^{m+n}, X\right) \longleftrightarrow \stackrel{g_{m+n}}{\longleftarrow} C_{m+n} X \stackrel{\alpha_{m+n}}{\longrightarrow} \Omega^{m+n} \Sigma^{m+n} X
\end{gathered}
$$

In defining $\zeta$ on the right, we thought of $S^{m+n}$ as $S^{n} \wedge S^{m}$ in order to have consistency with the $n$-fold loop map $\eta[n, m+n]: \Omega^{n} \Sigma^{n} X \rightarrow \Omega^{m+n} \Sigma^{m+n} X$. In turn, this motivated our switching $Z$ past $Y$ in Definition 6.2(i). Our Segal maps land in $\Omega^{n q} \sum^{n q} X^{[q]}$. When we embedded this space in the " $t$-fold loop" space $\Omega^{n q+t} \sum^{n q+t}$ in the introduction, it was the last $t$ loop coordinates we had in mind. On this 
understanding, the $t$-fold loop extension of $s_{q}$ is the bottom composite in the homotopy commutative diagram:

$$
\begin{gathered}
C\left(R^{t}, D_{q}\left(R^{n}, X\right)\right) \stackrel{C\left(1, s_{q}\right)}{\longrightarrow} C\left(R^{t}, C\left(R^{n q}, X^{[q]}\right)\right) \stackrel{\zeta}{\longrightarrow} C\left(R^{n q+t}, X^{[q]}\right) \\
\beta_{t} \downarrow \\
\left.\Omega^{t} \Sigma^{t} D_{q}\left(R^{n}, X\right) \stackrel{\Omega^{t} \Sigma^{t}\left(\beta_{n q} s_{q}\right)}{\longrightarrow} \Omega_{n q+t}\right) \\
\Omega^{t} \Sigma^{t} \Omega^{n q} \Sigma^{n q} X^{[q]} \stackrel{\zeta}{\longrightarrow} \Omega^{n q+t} \Sigma^{n q+t} X^{[q]}
\end{gathered}
$$

When convenient, we shall continue to write $s_{q}$ for any of its displayed composite variants.

Again, we could define partial power maps for general $\Sigma$-free separated coefficient systems, but we prefer to restrict attention to configuration space systems.

Definition 6.4. Define the $q$ th partial power map

$$
k_{q}: C(Y, X) \rightarrow C\left(F(Y, q), X^{[q]}\right)
$$

by $k_{q}\langle A, f\rangle=\left\langle F(A, q), f^{[q]} \mid F(A, q)\right\rangle$; compare Definition 6.1. Observe that the actual $q$ th power map

$$
p_{q}: C(Y, X) \stackrel{\Delta}{\rightarrow} C(Y, X)^{q} \stackrel{\otimes}{\rightarrow} C\left(Y^{q}, X^{[q]}\right)
$$

is given by the analogous formula $p_{q}\langle A, f\rangle=\left\langle A^{q}, f^{[q]}\right\rangle$. That is, $k_{q}$ results from $p_{q}$ by deletion of all points of $Y^{q}$ with repeated entries. By convention, $k_{0}$ and $p_{0}$ are constant at $1 \in S^{0}$. Clearly $k_{1}$ and $p_{1}$ are both the identity map; $k_{q}$ sends filtration $r$ to filtration $r ! /(r-q)$ ! while $p_{q}$ sends filtration $r$ to filtration $r^{q}$.

The following result is the combinatorial core of part (3) of Theorem $\mathrm{C}$ and thus of our proof of the Kahn-Priddy theorem. Its proof is trivial in view of the explicit set theoretical description of all the relevant maps.

PROPOSITION 6.5. The following diagram is commutative:

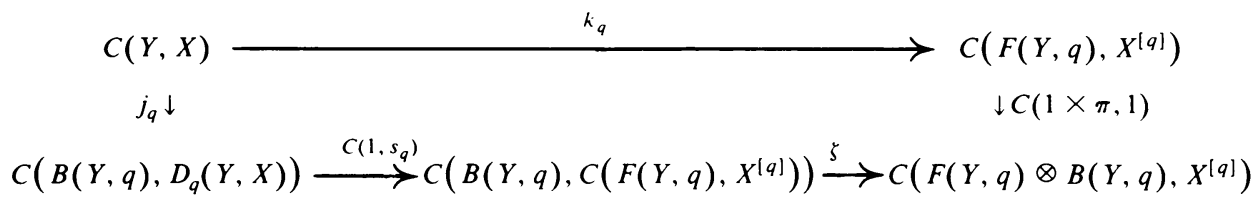

Upon embedding $F(Y, q)$ in $Y^{q}$, we can transform $C(1 \times \pi, 1)$ into a more convenient map in the cases of interest.

LEMMA 6.6. Let $\iota: F\left(R^{n}, q\right) \rightarrow R^{n q}$ be the inclusion and define $\lambda_{q}: R^{n q} \rightarrow R^{n q} \times$ $B\left(R^{n}, q\right)$ by $\lambda_{q}(y)=\left(y, b_{q}\right)$ for any chosen $b_{q} \in B\left(R^{n}, q\right)$. Then the following diagram is homotopy commutative:

$$
\begin{array}{ccc}
C\left(F\left(R^{n}, q\right), X^{[q]}\right) & C(\iota, 1) & C\left(R^{n q}, X^{[q]}\right) \\
C(1 \times \pi, 1) \downarrow & & \downarrow C\left(\lambda_{q}, 1\right) \\
C\left(F(Y, q) \times B(Y, q), X^{[q]}\right) & \stackrel{C(\llcorner\times 1,1)}{\rightarrow} & C\left(R^{n q} \times B\left(R^{n}, q\right), X^{[q]}\right)
\end{array}
$$


Proof. Choose an embedding $e: R^{n q} \rightarrow F\left(R^{n}, q\right)$ such that $\iota e$ is isotopic to the identity and $\pi e(0)=b_{q}$. Observe that the trapezoid commutes and the two triangles homotopy commute in the diagram:

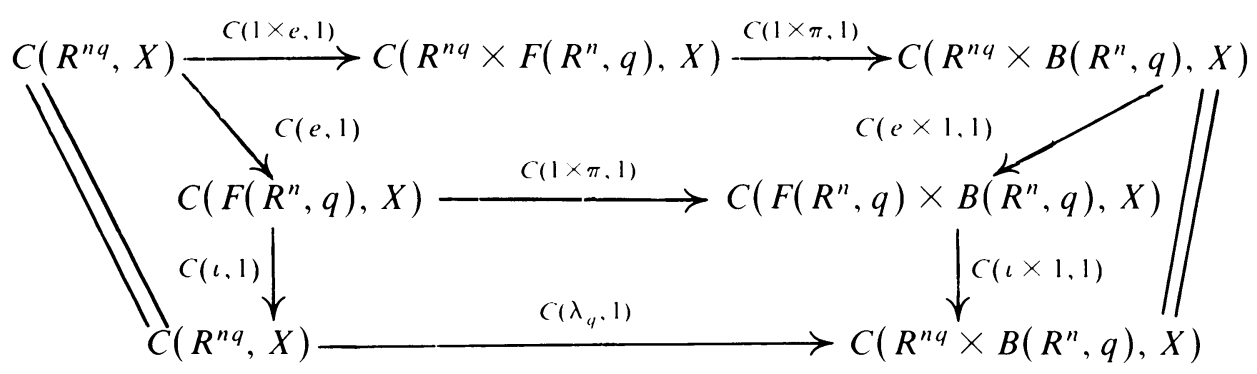

Since $\pi e$ is clearly homotopic through injections to $\lambda_{q}$, the top composite is homotopic to $C\left(\lambda_{q}, 1\right)$ and the conclusion follows.

7. Multiplicative properties of the maps $s_{q}$ and $k_{q}$. Here we complete the proof of Theorem B and of parts (1), (2) and (4) of Theorem C. We begin on the combinatorial level, retaining the notations of the previous section. Note that

$$
\oplus: C(Y, X) \times C(Z, W) \rightarrow C(Y \amalg Z, X \vee W)
$$

is given by $\langle A, f\rangle \oplus\langle B, g\rangle=\langle A \amalg B, f \vee g\rangle$, while

$$
\otimes: C(Y, X) \times C(Z, W) \rightarrow C(Y \times Z, X \wedge W)
$$

is given by $\langle A, f\rangle \otimes\langle B, g\rangle=\langle A \times B, f \wedge g\rangle$.

Throughout this section, let $r=p+q$. Recall from the introduction that $S_{p, q}$ denotes the set of $(p, q)$-shuffles in $\Sigma_{r}$. We need some more combinatorial maps.

Definition 7.1. Define maps

$$
\omega_{p, q}: C\left(F(Y, p) \times F(Z, q), X^{[p]} \wedge W^{[q]}\right) \rightarrow C\left(F(Y \amalg Z, r),(X \vee W)^{[r]}\right)
$$

and a further map, the formal sum of the $\omega_{p, q}$,

$$
\omega_{r}: \underset{p+q=r}{X} C\left(F(Y, p) \times F(Z, q), X^{[p]} \wedge W^{[q]}\right) \rightarrow C\left(F(Y \amalg Z, r),(X \vee W)^{[r]}\right)
$$

as follows. Regard $F(Y, p) \times F(Z, q)$ as a subspace of $F(Y \amalg Z, r)$ via the inclusions of $Y$ and $Z$ in $Y \amalg Z$ and regard $X^{[p]} \wedge W^{[q]}$ as a subspace of $(X \vee W)^{[r]}$ via the inclusions of $X$ and $W$ in $X \vee W$. For $A_{p, q} \subset F(Y, p) \times F(Z, q)$ and $f_{p, q}: A_{p, q} \rightarrow$ $X^{[p]} \wedge W^{[q]}$, define

$$
\omega_{p, q}\left\langle A_{p, q}, f_{p, q}\right\rangle=\left\langle A_{p, q} \cdot S_{p, q}, g_{p, q}\right\rangle,
$$

where $g_{p, q}(a \sigma)=\sigma^{-1} f_{p, q}(a)$ for $a \in A_{p, q}$ and $\sigma \in S_{p, q}$, and

$$
\omega_{r}\left(\underset{p+q=r}{\chi}\left\langle A_{p, q}, f_{p, q}\right\rangle\right)=\left\langle\coprod_{p+q=r} A_{p, q} \cdot S_{p, q}, \underset{p+q=r}{\vee} g_{p, q}\right\rangle .
$$

Write $\otimes \circ\left(s_{p} \wedge s_{q}\right)=s_{p} \otimes s_{q}$ and $\otimes \circ\left(k_{p} \times k_{q}\right)=k_{p} \otimes k_{q}$. Then easy computations give the following result. 
LEMMA 7.2. The following diagrams commute:

$$
\begin{array}{ccc}
D_{p}(Y, X) \wedge D_{q}(Z, W) & \stackrel{\oplus}{\rightarrow} & D_{r}(Y \amalg Z, X \vee W) \\
s_{p} \otimes s_{q} \downarrow & & \downarrow s_{r} \\
C\left(F(Y, p) \times F(Z, q), X^{[p]} \wedge W^{[q]}\right) & \stackrel{\omega_{p . q}}{\rightarrow} & C\left(F(Y \amalg Z, r),(X \vee W)^{[r]}\right)
\end{array}
$$

and

$$
\begin{array}{ccc}
C(Y, X) \times C(Z, W) & \stackrel{\oplus}{\rightarrow} & C(Y \amalg Z, X \vee W) \\
\times_{p+q=r} k_{p} \otimes k_{q} \downarrow & & \downarrow k_{r} \\
\underset{p+q=r}{X} C\left(F(Y, p) \times F(Z, q), X^{[p]} \wedge W^{[q]}\right) & \stackrel{\omega_{r}}{\rightarrow} & C\left(F(Y \amalg Z, r),(X \vee W)^{[r]}\right)
\end{array}
$$

Set $X=W$ and apply the folding map $\nabla: X \vee X \rightarrow X$, set $Y=Z$ and apply a good injection $i_{1}+i_{2}: Y \amalg Y \rightarrow Y$, and replace configuration spaces by Cartesian powers, all without change of notation. By the evident naturality of $s_{r}$ and $k_{r}$ and by the fact that $\omega_{p, q}$ and $\omega_{r}$ can be defined similarly and compatibly with configuration spaces replaced by Cartesian powers, the lemma implies the commutative diagrams:

$$
\begin{array}{ccc}
D_{p}(Y, X) \wedge D_{q}(Y, X) & \stackrel{\oplus}{\rightarrow} & D_{r}(Y, X) \\
s_{p} \otimes s_{q} \downarrow & & \downarrow s_{r} \\
C\left(Y^{r}, X^{[r]}\right) & \stackrel{\omega_{p . q}}{\rightarrow} & C\left(Y^{r}, X^{[r]}\right)
\end{array}
$$

and

$$
\begin{array}{ccc}
C(Y, X) \times C(Y, X) & \stackrel{\oplus}{\rightarrow} & C(Y, X) \\
\times_{p+q=r} k_{p} \otimes k_{q} \downarrow & & \downarrow k_{r} \\
\underset{p+q=r}{\times} C\left(Y^{r}, X^{[r]}\right) & \stackrel{\omega_{r}}{\rightarrow} & C\left(Y^{r}, X^{[r]}\right)
\end{array}
$$

Here, for $A_{p, q}=\{(\gamma, \delta)\} \subset Y^{p} \times Y^{q}$ and $f_{p, q}: A_{p, q} \rightarrow X^{[r]}$, we have

$$
\omega_{r}\left(\underset{p+q=r}{X}\left\langle A_{p, q}, f_{p, q}\right\rangle\right)=\left\langle\coprod_{p+q=r}\left(i_{1}^{p} \times i_{2}^{q}\right)\left(A_{p, q}\right) \circ S_{p, q}, g\right\rangle,
$$

where $g\left(\left(i_{1}^{p} \gamma, i \frac{q}{2} \delta\right) \sigma\right)=\sigma^{-1} f_{p, q}(\gamma, \delta)$. In practice, this formal sum $\omega_{r}$ and its summands $\omega_{p, q}$ are homotopic to actual sums.

LEMMA 7.3. If $Y=R^{n}, n \geqslant 2$, then $\omega_{r}$ is homotopic to the composite

$$
\underset{p+q=r}{\chi} C\left(R^{n r}, X^{[r]}\right) \stackrel{\times_{p+q=r} \omega_{p, q}}{\rightarrow} \underset{p+q=r}{X} C\left(R^{n r}, X^{[r]}\right) \stackrel{\oplus}{\rightarrow} C\left(R^{n r}, X^{[r]}\right)
$$


and $\omega_{p, q}$ is homotopic to the composite

$$
C\left(R^{n r}, X^{[r]}\right) \stackrel{\times_{\sigma} C\left(\sigma, \Sigma^{-1}\right)}{\rightarrow} \underset{\sigma \in S_{p, q}}{X} C\left(R^{n r}, X^{[r]}\right) \stackrel{\oplus}{\rightarrow} C\left(R^{n r}, X^{[r]}\right) .
$$

Proof. This is closely analogous to Lemmas 5.2 and 5.3, and a slight variant of the proof of the former leads to the homotopies required here.

Combining results, we obtain the following combinatorial version of parts (4) of Theorems B and C.

Proposition 7.4. Let $n \geqslant 2$. Then the following diagrams are homotopy commutative:

$$
\begin{gathered}
D_{p}\left(R^{n}, X\right) \wedge D_{q}\left(R^{n}, X\right) \stackrel{\oplus}{\longrightarrow} D_{r}\left(R^{n}, X\right) \\
s_{p} \otimes s_{q} \downarrow \\
\qquad\left(R^{n r}, X^{[r]}\right) \longrightarrow \stackrel{\Sigma_{\sigma \in S_{p, q}} C\left(\sigma, \sigma^{-1}\right)}{\longrightarrow} C\left(R^{n r}, X^{[r]}\right)
\end{gathered}
$$

and

$$
\begin{array}{cc}
C\left(R^{n}, X\right) \otimes C\left(R^{n}, X\right) \stackrel{\oplus}{\longrightarrow} C\left(R^{n}, X\right) \\
\quad \times_{p+q=r} k_{p} \otimes k_{q} \downarrow & \downarrow k_{r} \\
\underset{p+q=r}{\times} C\left(R^{n r}, X^{[r]}\right) \stackrel{\Sigma_{p+q=r} \Sigma_{\sigma \in S_{p . q}} C\left(\sigma, \sigma^{-1}\right)}{\longrightarrow} C\left(R^{n r}, X^{[r]}\right)
\end{array}
$$

Note that $C\left(\sigma, \sigma^{-1}\right)=C\left(1, \sigma^{-1}\right) C(\sigma, 1)$. We shall see in a moment that $\beta_{n r} C(\sigma, 1)$ $\simeq \tilde{\sigma} \beta_{n r}$, where $\tilde{\sigma}: \Omega^{n r} \sum^{n r} X \rightarrow \Omega^{n r} \sum^{n r} X$ is the conjugation homeomorphism specified in the introduction. This will complete the proof of part (4) of Theorem B. To complete the proof of part (4) of Theorem C, we use the following observation.

Proposition 7.5. For $n \geqslant 2$, the composite maps

$$
C\left(R^{n p}, X^{[p]}\right) \wedge C\left(R^{n q}, X^{[q]}\right) \stackrel{\otimes}{\rightarrow} C\left(R^{n r}, X^{[r]}\right) \stackrel{\Sigma_{\sigma \in S_{p . q}} C\left(\sigma, \sigma^{-1}\right)}{\longrightarrow} C\left(R^{n r}, X^{[r]}\right)
$$

and

$$
\Omega^{n p} \sum^{n p} X^{[p]} \wedge \Omega^{n q} \sum^{n q} X^{[q]} \stackrel{\wedge}{\rightarrow} \Omega^{n r} \sum^{n r} X^{[r]} \stackrel{\Sigma_{\sigma \in S_{p, q} \hat{\sigma}}}{\longrightarrow} \Omega^{n r} \sum^{n r} X^{[r]}
$$

are homotopy bilinear and constitute homotopy unital, associative, and commutative systems of pairings. They therefore give $X_{q \geqslant 0} C\left(R^{n q}, X^{[q]}\right)$ and $X_{q \geqslant 0} \Omega^{n q} \Sigma^{n q} X^{[q]}$ structures of $H$-semiring spaces such that $X_{q \geqslant 0} \beta_{n q}$ is a map of $H$-semiring spaces.

Proof. Homotopy bilinearity is clear since $C\left(\sigma, \sigma^{-1}\right)$ and $\hat{\sigma}$ are $H$-maps. The rest follows easily from standard unity, associativity, and commutativity properties of 
shuffle permutations. For commutativity, one should note that

$$
\otimes \circ \tau \simeq C\left(\tau_{m, n}, \tau\right) \circ \otimes: C\left(R^{m}, X\right) \wedge C\left(R^{n}, Y\right) \rightarrow C\left(R^{m+n}, X \wedge Y\right)
$$

and

$$
\wedge \circ \tau \simeq \tilde{\tau}_{m, n} \circ \wedge: \Omega^{m} \Sigma^{m} X \wedge \Omega^{n} \Sigma^{n} Y \rightarrow \Omega^{m+n} \Sigma^{m+n} X \wedge Y
$$

where the $\tau$ are transposition maps, $\tau_{m, n}$ is the twist homeomorphism $R^{m} \times R^{n} \rightarrow$ $R^{n} \times R^{m}$ or $S^{m} \wedge S^{n} \rightarrow S^{n} \wedge S^{m}$, and $\hat{\tau}_{m, n}=\left(\Omega^{m+n} \Sigma^{m+n} \tau\right) \circ \tilde{\tau}_{m, n}$.

Now Proposition 7.4 gives that $X_{q} k_{q}$ is an $H$-map into the unit space of $X_{q \geqslant 0} C\left(R^{n q}, X^{[q]}\right)$, while Corollary 1.7 gives that the unit space of $X_{q \geqslant 0} \Omega^{n q} \sum^{n q} X^{[q]}$ is grouplike. Therefore Proposition 1.2 gives an extension of $X_{q} k_{q}$ to a weak $H$-map from $\Omega^{n} \sum^{n} X$ to the latter unit space. The components of this $H$-map are the maps $k_{q}$ promised in Theorem $C$, and parts (1), (2) and (4) of that result are clear.

We must still verify that $\beta_{n r} C(\sigma, 1) \simeq \tilde{\sigma} \beta_{n r}$. We work more generally and consider a proper homeomorphism $\sigma: R^{n} \rightarrow R^{n}$; we also write $\sigma$ for its one-point compactification $S^{n} \rightarrow S^{n}$. If $\sigma$ is isotopic through homeomorphisms to the identity, then $C(\sigma, 1)$ and $\tilde{\sigma}$ are homotopic to the identity. Otherwise, $\sigma$ is isotopic through homeomorphisms to the map $\chi: R^{n} \rightarrow R^{n}$ which changes the sign of the first coordinate and leaves the remaining coordinates unchanged. (For $n=4$, we require $\sigma$ to be differentiable at some point to ensure this.) Thus, the following result implies the desired conclusion.

Lemma 7.6. For $n \geqslant 1, \beta_{n} \circ C(\chi, 1) \simeq \tilde{\chi} \circ \beta_{n}: C\left(R^{n}, X\right) \rightarrow \Omega^{n} \Sigma^{n} X$.

Proóf. Via $R \cong J$, we may consider $C(\chi, 1)$, where $\chi: J^{n} \rightarrow J^{n}$ is given by $\chi(s, t)=(1-s, t)$ for $s \in J$ and $t \in J^{n-1}$. Define a map $\chi: \bigodot_{n} \rightarrow \bigodot_{n}$ of coefficient systems by sending a little cube $c=c^{\prime} \times c^{\prime \prime}: J^{n} \rightarrow J^{n}$ to the little cube $\chi(c)=\chi\left(c^{\prime}\right)$ $\times c^{\prime \prime}$, where $\chi\left(c^{\prime}\right)(s)=1-c^{\prime}(1-s)$. Write $\hat{\chi}$ for the induced map $C_{n} X \rightarrow C_{n} X$. It is a simple matter to check that $C(\chi, 1) g_{n}=g_{n} \hat{\chi}$ and $\hat{\chi} \alpha_{n}=\alpha_{n} \hat{\chi}$.

8. The diagram relating $j_{q}, s_{q}$ and $k_{q}$. Here we complete the proof of part (3) of Theorem C. Recall the notations (5.a)-(5.d) and adopt the following analogs, where $t=t(n, q)$ :

$$
\begin{gathered}
\bar{C}(n, q, X)=C\left(R^{n q} \times B\left(R^{n}, q\right), X^{[q]}\right), \\
\bar{\gamma}_{n q+t}=\beta_{n q+t} \circ C\left(1 \times d_{q}, 1\right): \bar{C}(n, q, X) \rightarrow \Omega^{n q+t} \Sigma^{n q+t} X^{[q]}, \\
\bar{G}(n, q, X)=\Omega C\left(R^{n q-1} \times B\left(R^{n}, q\right), \Sigma X^{[q]}\right), \\
\bar{\delta}_{n q+t}=\beta_{n q-1+t} \circ C\left(1 \times d_{q}, 1\right): C\left(R^{n q-1} \times B\left(R^{n}, q\right), \Sigma X^{[q]}\right) \\
\rightarrow \Omega^{n q-1+t} \sum^{n q+t} X^{[q]} .
\end{gathered}
$$

By Theorem 5.6 and the sentence following it, we have a group completion $\beta: \bar{C}(n, q, X) \rightarrow \bar{G}(n, q, X)$ such that $\bar{\gamma}_{n q+t} \simeq\left(\Omega \bar{\delta}_{n q+t}\right) \circ \beta$. Consider the following diagram; we apologize for its size (and for writing $t$ ambiguously for the $t(n, q)$ ), but putting things together this way does seem to help keep track of the argument. 


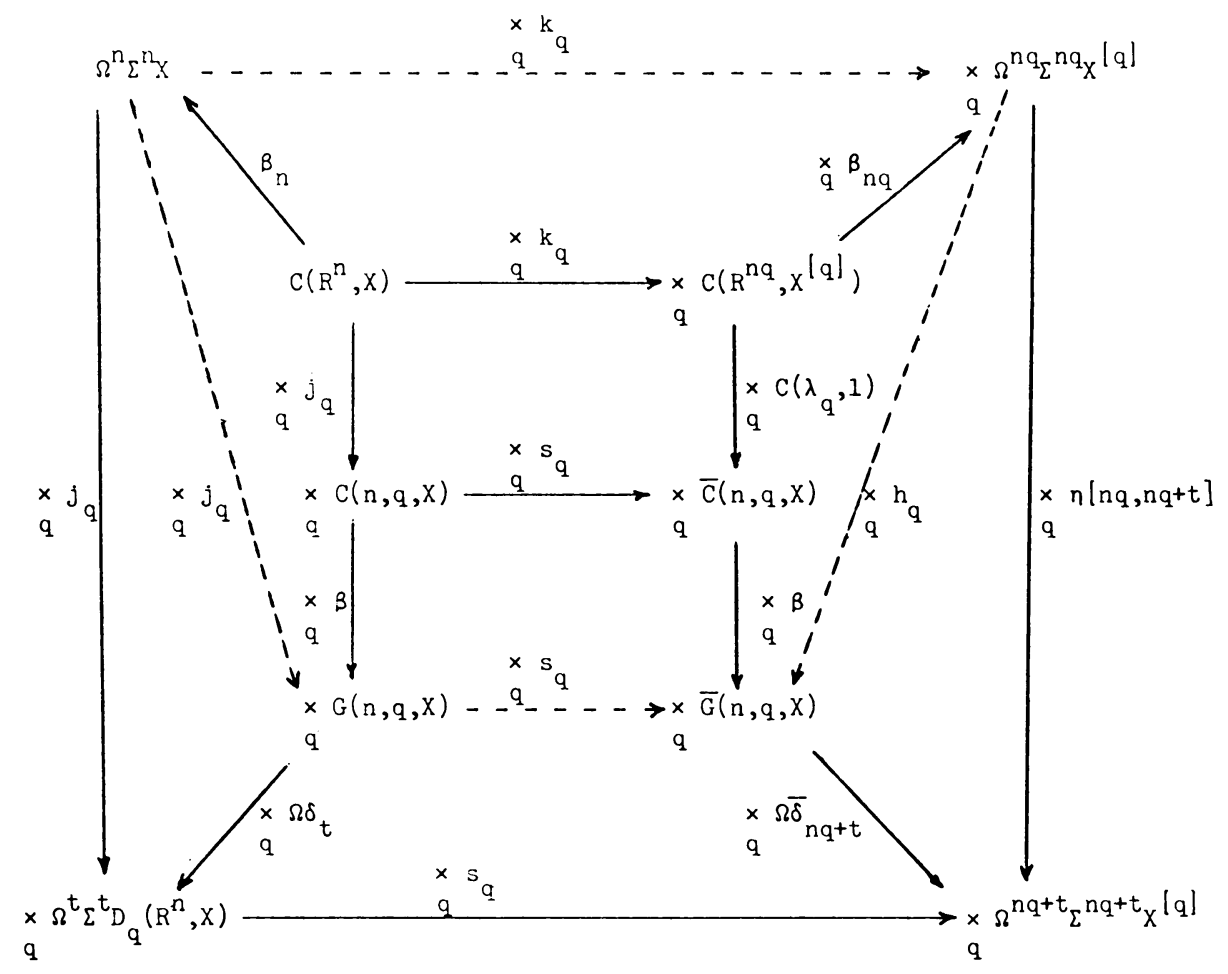

All dotted arrows were, or will be, obtained by use of the universal properties of group completions. The left trapezoid summarizes the construction in $\$ 5$ of the James maps used to prove Theorem A. The upper rectangle is the concatenation of the diagrams in Proposition 6.5 and Lemma 6.6, with $s_{q}$ and $k_{q}$ written for the resulting composite $H$-maps. With the dotted arrows $s_{q}$ erased, the lower central diagram is homotopy commutative by an easy, but lengthy, chase based on naturality and the diagram following Lemma 6.3. The dotted arrows $s_{q}$ are obtained by Proposition 1.2 so that $s_{q} \beta \stackrel{\mathrm{w}}{\simeq} \beta s_{q}$, and then the bottom trapezoid is weakly homotopy commutative by the uniqueness clause of that result. With the dotted arrows $h_{q}$ erased, the right trapezoid homotopy commutes, this being obvious once we note that we can choose $b_{q} \in B\left(R^{n}, q\right)$ and $d_{q}: B\left(R^{n}, q\right) \rightarrow R^{t}$ so that $d_{q}\left(b_{q}\right)=0$. Since $C\left(\lambda_{q}, 1\right)$ is certainly an $H$-map (see Lemma 5.5), Proposition 1.2 applies again to give dotted arrows $h_{q}$ making the two parts of the right trapezoid weakly homotopy commutative. Dotted arrows $k_{q}$ making the top trapezoid weakly homotopy commutative were obtained in the previous section.

To sum up, the entire diagram will be weakly homotopy commutative if $h_{q} k_{q} \stackrel{\mathrm{w}}{\simeq} s_{q} j_{q}$, and these two composites become weakly homotopic when composed with $\beta_{n}$. We showed in $\S \S 5$ and 7 that $\times_{q} C(n, q, X)$ and $\times_{q} C\left(R^{n q}, X^{[q]}\right)$ are $H$-semiring spaces such that $X_{q} j_{q}$ and $X_{q} k_{q}$ are $H$-maps into their unit spaces. We shall shortly prove the following result. 
Proposition 8.1. There are homotopy bilinear maps

$$
\bar{C}(n, p, X) \wedge \bar{C}(n, q, X) \rightarrow \bar{C}(n, p+q, X)
$$

which constitute a homotopy unital, associative, and commutative system of pairings such that the diagram

$$
\underset{q}{X} C(n, q, X) \stackrel{\times_{q} s_{q}}{\rightarrow} \underset{q}{X} \bar{C}(n, q, X) \stackrel{\times_{q} C\left(\lambda_{q}, 1\right)}{\leftarrow} \underset{q}{\times} C\left(R^{n q}, X^{[q]}\right)
$$

displays two maps of $H$-semiring spaces.

By Corollary 1.7, it will follow that $\times_{q} \bar{G}(n, q, X)$ is a weak $H$-ring space such that its unit space is grouplike and $X_{q} \beta$ is a map of weak $H$-semiring spaces. The uniqueness clause of Proposition 1.4 will apply to give that the dotted arrows $X_{q} s_{q}$ and $X_{q} h_{q}$ are also maps of weak $H$-semiring spaces. Finally, the uniqueness clause of Proposition 1.2 will then give $X_{q} h_{q} k_{q} \stackrel{\mathrm{w}}{\simeq} \times_{q} s_{q} j_{q}$.

Before proving the proposition, we interpolate the following naturality diagrams relating the maps $\zeta$ of Definition 6.2(i) to sums and products; the first has already been used implicitly in our assertion that the maps $s_{q}$ of the proposition are additive $H$-maps:

$$
\begin{array}{ccc}
C(Y, C(Z, X)) \times C\left(Y^{\prime}, C\left(Z^{\prime}, X^{\prime}\right)\right) & \stackrel{\oplus}{\rightarrow} & C\left(Y \amalg Y^{\prime}, C(Z, X) \vee C\left(Z^{\prime}, X^{\prime}\right)\right) \\
\zeta \times \zeta \downarrow & & \\
C(Z \times Y, X) \times C\left(Z^{\prime} \times Y^{\prime}, X^{\prime}\right) & & C\left(Y \amalg Y^{\prime}, C\left(Z \amalg Z^{\prime}, X \vee X^{\prime}\right)\right) \\
\oplus \downarrow & & \\
\downarrow\left((Z \times Y) \amalg\left(Z^{\prime} \times Y^{\prime}\right), X \vee X^{\prime}\right) & \rightarrow & C\left(\left(Z \amalg Z^{\prime}\right) \times\left(Y \amalg Y^{\prime}\right), X \vee X^{\prime}\right)
\end{array}
$$

where evident inclusions give the unlabeled arrows, and

$$
\begin{array}{cc}
C(Y, C(Z, X)) \times C\left(Y^{\prime}, C\left(Z^{\prime}, X^{\prime}\right)\right) & \otimes \\
\zeta \times \zeta \downarrow & C\left(Y \times Y^{\prime}, C(Z, X) \wedge C\left(Z^{\prime}, X^{\prime}\right)\right) \\
C(Z \times Y, X) \times C\left(Z^{\prime} \times Y^{\prime}, X^{\prime}\right) & \\
\otimes \downarrow & C\left(Y \times Y^{\prime}, C\left(Z \times Z^{\prime}, X \wedge X^{\prime}\right)\right) \\
\downarrow & \downarrow \zeta \\
C\left(Z \times Y \times Z^{\prime} \times Y^{\prime}, X \wedge X^{\prime}\right) \stackrel{C(1 \times \tau \times 1,1)}{\longrightarrow} C\left(Z \times Z^{\prime} \times Y \times Y^{\prime}, X \wedge X^{\prime}\right)
\end{array}
$$

where $\tau$ denotes the transposition map.

To prove the proposition, note that the maps $\omega_{p, q}$ of Definition 7.1 can be defined equally well with all left-hand variables crossed with a given space $B$ with trivial action by $\Sigma_{r}$. The following diagram then commutes:

$$
\begin{array}{ccc}
C\left(B, C\left(F(Y, p) \times F(Z, q), X^{[p]} \wedge W^{[q]}\right)\right) & \stackrel{\zeta}{\rightarrow} & C\left(F(Y, p) \times F(Z, q) \times B, X^{[p]} \wedge W^{[q]}\right) \\
C\left(1, \omega_{p, q}\right) \downarrow & & \downarrow \omega_{p, q} \\
C\left(B, C\left(F(Y \amalg Z, r),(X \vee W)^{[r]}\right)\right) & \stackrel{\zeta}{\rightarrow} & C\left(F(Y \amalg Z, r) \times B,(X \vee W)^{[r]}\right)
\end{array}
$$


When $Y=Z=R^{n}$, Lemma 7.3 generalizes to evaluate the resulting internalized maps $\omega_{p, q}$ as sums $\Sigma_{\sigma \in S_{p, q}} C\left(\sigma, \sigma^{-1}\right)$.

The multiplications promised in Proposition 8.1 are given by the dotted arrow composites in the diagrams

$$
\begin{aligned}
& \bar{C}(n, p, X) \wedge \bar{C}(n, q, X) \longrightarrow \stackrel{\otimes}{\longrightarrow} C\left(R^{n p} \times B\left(R^{n}, p\right) \times R^{n q} \times B\left(R^{n}, q\right), X^{[p]} \wedge X^{[q]}\right) \\
& \begin{array}{c}
\downarrow C(1 \times \tau \times 1,1) \\
\left.\qquad \begin{array}{c} 
\\
\vdots \\
\vdots
\end{array}(n, r, X) \longleftarrow R^{n r} \times B\left(R^{n}, p\right) \times B\left(R^{n}, q\right), X^{[r]}\right) \\
\downarrow C(1 \times \oplus, 1) \\
\Sigma_{o \in S_{p . q}} C\left(\sigma, \sigma^{-1}\right) \\
\bar{C}(n, r, X)=C\left(R^{n r} \times B\left(R^{n}, r\right), X^{[r]}\right)
\end{array}
\end{aligned}
$$

where $r=p+q$. Recalling Lemma 5.3 and the fact that $s_{q}$ in Proposition 8.1 denotes $\zeta \circ C\left(1, s_{q}\right)$, we see that Proposition 7.4 and the diagrams above give that $\times_{q} s_{q}$ is a map of $H$-semiring spaces. Composing these pairings with those of Proposition 7.5, we see that any choices of paths in $B(R, r)$ connecting $b_{p} \oplus b_{q}$ to $b_{r}$ determine homotopies which show that $\times_{q} C\left(\lambda_{q}, 1\right)$ is a map of $H$-semiring spaces.

9. Decomposition of the power maps. We prove Theorem $\mathrm{C}(5)$ and Theorem $\mathrm{D}$ here. Recall the notation above Theorem C. As there, for $\rho \in P_{m, q}$, define $\rho: F(Y, m) \rightarrow Y^{q}$ by

$$
\rho\left(y_{1}, \ldots, y_{m}\right)=\left(z_{1}, \ldots, z_{q}\right), \quad \text { where } z_{j}=y_{i} \text { if } j \in s_{i} .
$$

These maps $\rho$ partition $Y^{q}$ in the sense of the following result.

LEMMA 9.1. Regard $P_{m, q}$ as a discrete space and let

$$
\phi: \coprod_{1 \leqslant m \leqslant q} F(Y, m) \times P_{m, q} \rightarrow Y^{q}
$$

have $\rho$ th restriction the map $\rho$ just specified. Then $\phi$ is a continuous bijection.

An easy calculation gives the corresponding decomposition of the combinatorial power map $p_{q}$.

LEMMA 9.2. The following diagram is commutative:

$$
\begin{gathered}
C(Y, X) \stackrel{\times_{m} k_{m}}{\longrightarrow} \underset{m=1}{X} C\left(F(Y, m), X^{[m]}\right) \stackrel{\times_{m} \times_{\rho} C(1, \rho)}{\longrightarrow} \underset{m=1}{\underset{p^{\prime}}{\chi}} \underset{\rho \in P_{m, q}}{X} C\left(F(Y, m), X^{[q]}\right) \\
C\left(Y^{q}, X^{[q]}\right) \stackrel{C(\phi, 1)}{\longrightarrow} C\left(\coprod_{1 \leqslant m \leqslant q} F(Y, m) \times P_{m, q}, X^{[q]}\right)
\end{gathered}
$$

At this point, our usual device of including configuration spaces in Cartesian powers causes a bit of a problem since we obviously cannot extend our bijection $\phi$ to an injection $\amalg_{1 \leqslant m \leqslant q} Y^{m} \times P_{m, q} \rightarrow Y^{q}$. With $Y=R^{n}, n \geqslant 2$, we overcome this problem by use of an isotopy of $\phi$ which we now describe. 
Of course, $\phi$ is the restriction of a surjective map

$$
\bar{\phi}: \coprod_{1 \leqslant m \leqslant q} R^{n m} \times P_{m, q} \rightarrow R^{n q} .
$$

Choose an integer $a_{\rho}$ for each $\rho \in \amalg_{1 \leqslant m \leqslant q} P_{m, q}$, taking the $a_{\rho}$ to be distinct. Let $b_{\rho} \in R^{n m}$ be the element all of whose coordinates are $a_{\rho}$. Let $c=\sum_{m=1}^{q} c_{m q}$ and observe that $\left\{\bar{\phi}\left(b_{\rho}\right)\right\}$ is just a set of $c$ distinct points each of which is a distance at least 1 away from all the others in the usual Euclidean metric.

For $\rho \in P_{m, q}$, choose an isotopy $h_{t}^{\rho}: R^{n} \rightarrow R^{n}$ such that $h_{0}^{\rho}$ is the identity and $h_{1}^{\rho}$ takes image in the interior of the ball of radius $1 / q$ with center $\left(a_{\rho}, \ldots, a_{\rho}\right)$. Define a homotopy $\bar{\phi}_{t}$ of $\bar{\phi}$ by letting its $m$ th restriction be given by the formula

$$
\bar{\phi}_{t}\left(y_{1}, \ldots, y_{m}, \rho\right)=\bar{\phi}\left(h_{t}^{\rho}\left(y_{1}\right), \ldots, h_{t}^{\rho}\left(y_{m}\right)\right)
$$

for $y_{i} \in R^{n}$. Let $\psi=\bar{\phi}_{1}$ and observe that $\psi$ is an embedding. Since $\bar{\phi}_{t}$ restricts on $\amalg_{1 \leqslant m \leqslant q} F\left(R^{n}, m\right) \times P_{m, q}$ to an isotopy of $\phi$, we conclude from the previous lemma that the following diagram is homotopy commutative:

$$
\begin{aligned}
& C\left(R^{n}, X\right) \stackrel{\times_{m} k_{m}}{\longrightarrow} \underset{m=1}{\chi} C\left(R^{n m} \cdot X^{[m]}\right) \stackrel{\times_{m} \times_{\rho} C(1, \rho)}{\longrightarrow} \underset{m=1}{\chi} \underset{\rho \in P_{m, q}}{X} C\left(R^{n m}, X^{[q]}\right) \\
& p_{q} \downarrow \quad \downarrow \oplus \\
& C\left(R^{n q}, X^{[q]}\right) \longleftarrow C(\psi, 1) \quad C\left(\coprod_{1 \leqslant m \leqslant q} R^{n m} \times P_{m . q}, X^{[q]}\right)
\end{aligned}
$$

For each $m$ and $\rho$, the restriction of $\psi$ to $R^{n m} \times\{\rho\}$ is isotopic to the standard inclusion. (For $m=q$, there is just one $\rho$ and the corresponding restriction is isotopic to the identity.) By Lemma A.2 below, we conclude that $C(\psi, 1) \circ \oplus$ is homotopic to the composite

$$
{\underset{m=1}{\chi}}_{\rho \in P_{m, q}} C\left(R^{n m}, X\right) \stackrel{\times_{m} \times_{\rho} \eta[n m, n q]}{\longrightarrow} \underset{m=1}{\chi} \underset{\rho \in P_{m, q}}{\chi} C\left(R^{n q}, X^{[q]}\right) \stackrel{\oplus}{\rightarrow} C\left(R^{n q}, X^{[q]}\right),
$$

where $\eta[n m, n q]$ is induced by the standard inclusion $R^{n m} \subset R^{n q}$.

This proves Theorem $\mathrm{C}(5)$ on the combinatorial level. Of course, $\beta_{n q} p_{q} \simeq p_{q} \beta_{n}$, where $p_{q}$ on the right is the smash power. By the construction of the $k_{q}$, we also have

$$
\begin{aligned}
& \beta_{n q}\left(\sum_{m=1}^{q} \sum_{\rho \in P_{m, q}} \eta[n m, n q] \circ C(1, \rho) \circ k_{m}\right) \\
& \stackrel{\mathrm{w}}{\simeq} \sum_{m=1}^{q} \sum_{\rho \in P_{p . q}}\left(\eta[n m, n q] \circ \Omega^{n m} \Sigma^{n m} \rho \circ k_{m}\right) \beta_{n} .
\end{aligned}
$$

We shall apply our usual uniqueness trick to conclude that the smash power is weakly homotopic to the relevant sum. To justify this, we use the following analog of Lemma 7.2, in which $p_{p} \otimes p_{q}=\otimes \circ\left(p_{p} \times p_{q}\right)$. This is really just a disguised form of the binomial theorem. 
LEMMA 9.3. The following diagram commutes:

$$
\begin{array}{ccc}
C(Y, X) \times C(Z, W) & \stackrel{\oplus}{\rightarrow} & C(Y \amalg Z, X \vee W) \\
\times_{p+q=r} p_{p} \otimes p_{q} \downarrow & & \downarrow p_{r} \\
\underset{p+q=r}{X} C\left(Y^{p} \times Z^{q}, X^{[p]} \wedge W^{[q]}\right) & \stackrel{\omega_{r}}{\rightarrow} & C\left((Y \amalg Z)^{r},(X \vee W)^{r}\right)
\end{array}
$$

Internalizing and applying Lemma 7.3, we obtain the following analog of Proposition 7.4.

Proposition 9.4. Let $n \geqslant 2$. Then the following diagram is homotopy commutative:

$$
\begin{array}{cc}
C\left(R^{n}, X\right) \times C\left(R^{n}, X\right) \stackrel{\oplus}{\oplus} C\left(R^{n}, X\right) & \downarrow p_{r} \\
\times_{p+q=r} p_{p} \otimes p_{q} \downarrow & \\
\underset{p+q=r}{X} C\left(R^{n r}, X^{[r]}\right) \stackrel{\Sigma_{p+q=r} \Sigma_{\sigma \in S_{p, q}} C\left(\sigma, \sigma^{-1}\right)}{\longrightarrow} C\left(R^{n r}, X^{[r]}\right)
\end{array}
$$

Thus $X_{q} p_{q}$ is an $H$-map into the unit space of $X_{q} C\left(R^{n q}, X^{[q]}\right)$; compare Proposition 7.5. It is easy to check the corresponding version of the binomial theorem for the smash powers $p_{q}$. It takes more work to use the known multiplicative properties of the $k_{m}$ to deduce that

$$
\underset{q}{X}\left(\sum_{m=1}^{q} \sum_{\rho \in P_{m, q}}\left(\eta[n m, n q] \circ \Omega^{n m} \sum^{n m} \rho \circ k_{m}\right)\right)
$$

is also a weak $H$-map from $\Omega^{n} \sum^{n} X$ to the unit space of $X_{q} \Omega^{n q} \sum^{n q} X^{[q]}$. These verifications made, Proposition 1.2 applies to show that the last two maps are weakly homotopic.

To prove Theorem $\mathrm{D}$, we apply the following algebraic fact to $\times_{q}\left[A^{+}, \Omega^{n q} S^{n q}\right]$ regarded as a ring under componentwise loop sums and smash products. Note that this ring is not commutative if $n$ is odd; compare the proof of Proposition 7.5.

LEMMA 9.5. In a ring, the functions $f_{q}(x)$ specified by the implicit formulas $x^{q}=\sum_{m=1}^{q} c_{m, q} f_{m}(x)$ are given by the explicit formulas

$$
f_{q}(x)=x(x-1) \cdots(x-q+1) .
$$

Proof. If $Y$ is a set with $r$ elements, the bijection $\phi$ of Lemma 9.1 and the fact that $F(Y, m)$ has $r ! /(r-m)$ ! elements imply the formula $r^{q}=\sum_{m=1}^{q} c_{m, q} r ! /(r-m)$ ! for positive integers $r$. This implies the conclusion for the free ring $(=$ polynomial ring) on one variable, and the conclusion follows, in general, by universality.

10. Multiplicative properties of some more James maps. In [7], we constructed stable splittings of certain spaces

$$
\bar{C} X=\operatorname{colim} \bigodot_{r} \times_{\Sigma_{r}} X^{r}
$$


associated to directed coefficient systems $C$. Here $C$ is directed if there are sub$\Sigma_{r}$-spaces $\hat{\bigcup}_{r+1}$ of $\bigcup_{r+1}$ and $\Sigma_{r}$-equivariant homotopy equivalences $\lambda_{r}: \bigodot_{r} \rightarrow \hat{\bigcup}_{r+1}$ which satisfy the following properties:

(i) The inclusion $\bigcap_{i \in s} \hat{\varrho}_{r+1} \tau^{i} \rightarrow \mathcal{C}_{r+1}$ is a $\Sigma_{s}$-cofibration, where $\tau \in \Sigma_{r+1}$ is the cyclic permutation $(1,2, \ldots, r+1)$, $s$ is any subset of $\{0,1, \ldots, r\}$, and $\Sigma_{s} \subset \Sigma_{r+1}$ is the group of permutations $\sigma$ such that if $i \in s$ and $\tau^{i} \sigma \in \Sigma_{r-1} \tau^{j}$, then $j \in s$.

(ii) $\phi_{r} \lambda_{r}=1: \bigodot_{r} \rightarrow \bigodot_{r}$, where $\phi_{r}: \mathbf{r} \rightarrow \mathbf{r}+1$, is the injection specified by $\phi_{r}(i)=i$ for $0 \leqslant i \leqslant r$.

(iii) $\omega \lambda_{r}$ takes values in $\hat{\bigcup}_{q}$ for any ordered injection $\omega: \mathbf{q} \rightarrow \mathbf{r}+\mathbf{1}$ such that $\omega(q)=r+1$.

The cofibration condition (i) was misstated in [7] but, as stated and used there, it is implied by the following two conditions.

(ia) For $c \in \hat{\mathcal{C}}_{r+1}$ and $\sigma \in \Sigma_{r+1}, c \sigma \in \hat{\mathrm{e}}_{r+1}$ if and only if $\sigma \in \Sigma_{r}$.

(ib) The inclusion of $\hat{\mathcal{C}}_{r+1}$ in $\mathcal{C}_{r+1}$ is a $\Sigma_{r}$-cofibration.

Given such a structure on $C$, the maps of the colimit system used to define $\bar{C} X$ are defined to be

$$
\zeta_{r}=\lambda_{r} \times \phi_{r}: e_{r} \times_{\Sigma_{r}} X^{r} \rightarrow e_{r+1} \times_{\Sigma_{r+1}} X^{r+1},
$$

where $\phi_{r}(y)=(y, *)$. The cofibre of $\zeta_{r}$ is equivalent to the quotient space

$$
\begin{aligned}
\bar{D}_{r+1}(\mathcal{C}, X) & =\mathcal{e}_{r+1} \times \Sigma_{\Sigma_{r+1}} X^{r+1} / \hat{e}_{r+1} \times \Sigma_{\Sigma_{r}} X^{r} \\
& =\left[\mathcal{C}_{r+1} \times X^{r+1} /\left(\hat{e}_{r+1} \times X^{r}\right) \Sigma_{r+1}\right] / \Sigma_{r+1}
\end{aligned}
$$

Again, by convention, we set $\bar{D}_{0}(\bigodot, X)=S^{0}$.

For a James system $\left\{\xi_{q, r}\right\}: \bigodot \rightarrow C^{\prime}$, precisely the same formula as in $\S 3$ gives a James map $\bar{j}_{q}: \bar{C} X \rightarrow C^{\prime} \bar{D}_{q}(e, X)$. We use the following definition to relate these James maps to sums.

Definition 10.1. Let $\sigma_{p, q} \in \Sigma_{p+q+1}$ fix the first $p$ letters and permute the $(p+1)$ st letter past the remaining $q$ letters. Let $Q, \mathscr{B}$ and $\mathcal{C}$ be directed coefficient systems. A sum $\bigoplus:(\mathscr{Q}, \mathfrak{B}) \rightarrow \mathcal{C}$ is directed if the following conditions hold:

(i) The following maps have images contained in $\hat{\bigodot}_{p+q}$ :

$$
\hat{\mathscr{Q}}_{p} \times \mathscr{B}_{q} \stackrel{\oplus}{\rightarrow} \bigodot_{p+q} \stackrel{\sigma_{p-i}, q}{\rightarrow} \bigodot_{p+q} \text { and } \mathcal{Q}_{p} \times \hat{\mathscr{B}}_{q} \stackrel{\oplus}{\rightarrow} \bigodot_{p+q} .
$$

(ii) The following diagrams are commutative:

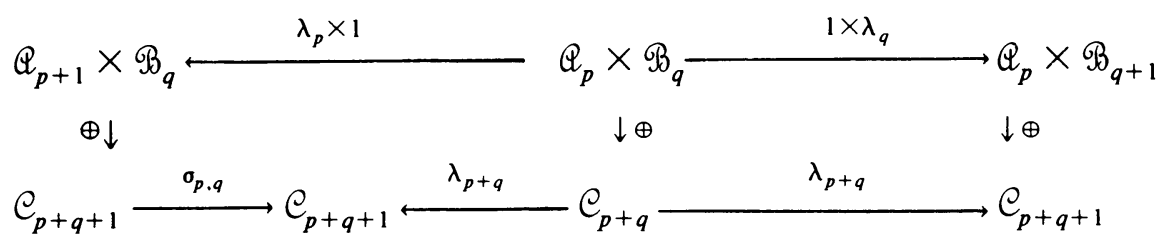

The sum $\oplus$ is weakly directed if (i) holds and the diagrams of (ii) commute up to $\Sigma_{p} \times \Sigma_{q}$-homotopy, where $\Sigma_{p} \times \Sigma_{q}$ is embedded in $\Sigma_{p+q+1}$ via $(\sigma, \tau) \rightarrow \sigma \oplus \tau \oplus 1$.

We have the following analog of Lemma 2.3, the last statement of which is verified by use of the natural equivalence $\operatorname{Tel}\left(e_{r} \times_{\Sigma_{r}} X^{r}\right) \rightarrow \bar{C} X$. 
LEMma 10.2. For a directed sum $\oplus:(Q, \Re) \rightarrow C$ and based spaces $X$ and $Y$, there is a natural induced map

$$
\bigoplus: \bar{A} X \times \bar{B} Y \rightarrow \bar{C}(X \vee Y)
$$

whose restriction $\left(\mathscr{Q}_{p} \times_{\Sigma_{p}} X^{p}\right) \times\left(\mathscr{B}_{q} \times_{\Sigma_{q}} Y^{q}\right) \rightarrow \mathcal{C}_{r} \times_{\Sigma_{r}}(X \vee Y)^{r}, r=p+q$, passes to quotients to give

$$
\oplus: \bar{D}_{p}(Q, X) \wedge \bar{D}_{q}(\Re, Y) \rightarrow \bar{D}_{r}(X \vee Y)
$$

If $\oplus$ is only weakly directed, then the first map still exists but is only determined up to weak homotopy.

When $X=Y$, we continue to write $\oplus$ for internalized sums.

Since James maps and sums are defined by precisely the same formulas here as earlier in the paper, the following analog of Proposition 3.4 admits the same proof, telescopes again being used for the last statement.

Proposition 10.3. Given a pairing of James systems as in Definition 3.3, where $\oplus$ : $(Q, \mathscr{B}) \rightarrow \mathcal{C}$ is directed, the following diagram commutes for each $r \geqslant 0$ :

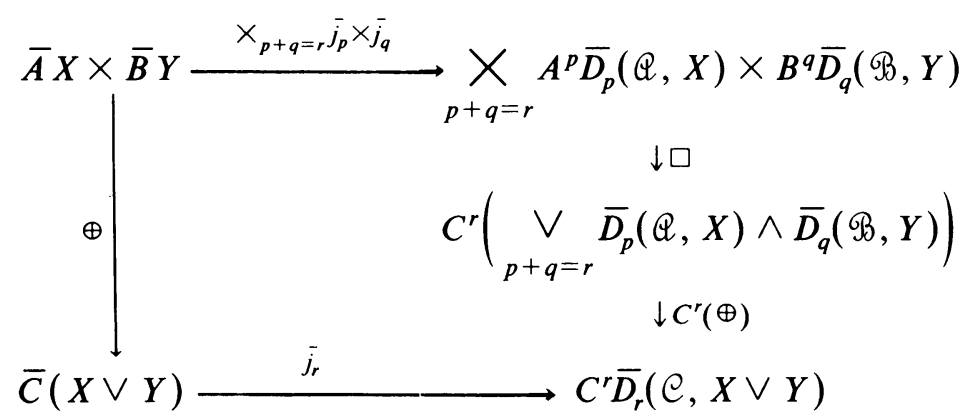

If $\oplus$ is only weakly directed, the diagram still commutes up to weak homotopy.

Via Example 3.5, we obtain the following analog of Theorem 3.6; compare [7, 2.5].

THEOREM 10.4. Let $\boxplus:(\mathscr{Q}, \mathscr{B}) \rightarrow \mathcal{C}$ be a weakly directed sum. For any based spaces $X$ and $Y$ and Abelian group $G$, the following is a natural commutative diagram in which the horizontal arrows are isomorphisms:

$$
\begin{gathered}
\tilde{H}_{*}(\bar{A} X \times \bar{B} Y ; G) \stackrel{\Sigma_{r>1} \Sigma_{p+q=r}\left(\overline{j_{p}} \wedge \bar{j}_{q}\right)_{*}}{\longrightarrow} \sum_{r \geqslant 1} \sum_{p+q=r} \tilde{H}_{*}\left(\bar{D}_{p}(Q, X) \wedge \bar{D}_{q}(\Re, Y) ; G\right) \\
\oplus_{*} \downarrow \\
\tilde{H}_{*}(\bar{C}(X \vee Y) ; G) \stackrel{\Sigma_{r>1}\left(\bar{j}_{r}\right)_{*}}{\longrightarrow} \sum_{r \geqslant 1} \tilde{H}_{*}\left(\bar{D}_{r}(\mathcal{C}, X \vee Y) ; G\right)
\end{gathered}
$$

COROLlARY 10.5. For any commutative coefficient ring, the pairing

$$
\bigoplus_{*}: \tilde{H}_{*} \bar{A} X \otimes \tilde{H}_{*} \bar{B} X \rightarrow \tilde{H}_{*} \bar{C} X
$$

can be computed as the sum of the quotient pairings

$$
\bigoplus_{*}: \tilde{H}_{*} \bar{D}_{p}(\Theta, X) \otimes \tilde{H}_{*} \bar{D}_{q}(\mathscr{B}, X) \rightarrow \tilde{H}_{*} \bar{D}_{p+q}(\bigodot, X) .
$$


For example, the sum $\oplus:(\mathfrak{N}, \mathfrak{N}) \rightarrow \mathfrak{N}$ is directed. Here $\bar{D}_{p}(\mathfrak{N}, X)$ is the quotient $\left(X^{p} / \Sigma_{p}\right) /\left(X^{p-1} / \Sigma_{p-1}\right)$ of unreduced symmetric powers.

Before proceeding to the analog of Theorem 4.6, we consider some examples and counterexamples.

EXAMPLES 10.6. The product $C \times C^{\prime}$ of directed coefficient systems $C$ and $C^{\prime}$ is directed by the spaces $\hat{\bigcup}_{r+1} \times \hat{\bigcup}_{r+1}^{\prime}$ and maps $\lambda_{r} \times \lambda_{r}^{\prime}$. The product of two (weakly) directed sums is (weakly) directed.

The most interesting examples in [7] were the coefficient systems $e\left(R^{n}\right)$. Unfortunately, the present theory does not quite work for them.

COUNTEREXAMPLE 10.7. Let $Y=R \times Z$ for a nondegenerately based space $Z$. By [7, 1.4], $\mathcal{C}(Y)$ is directed with respect to the spaces

$$
\hat{F}(Y, r+1)=\left\{\left\langle\left(t_{1}, z_{1}\right), \ldots,\left(t_{r}, z_{r}\right),(t, *)\right\rangle \mid t \geqslant 1+\max t_{i}\right\}
$$

and maps $\lambda_{r}: F(Y, r) \rightarrow F(Y, r+1)$ specified by

$$
\lambda_{r}\left\langle\left(t_{1}, z_{1}\right), \ldots,\left(t_{r}, z_{r}\right)\right\rangle=\left\langle\left(t_{1}, z_{1}\right), \ldots,\left(t_{r}, z_{r}\right),\left(1+\max t_{i}, *\right)\right\rangle .
$$

Use a good embedding $R \amalg R \rightarrow R$ to give $\mathcal{C}(Y)$ an internal sum $\oplus$. If $Z=R \times Z^{\prime}$, then it is easy to verify, by use of Lemma 2.6, that the diagrams of Definition 10.1(ii) commute up to $\Sigma_{p} \times \Sigma_{q}$-homotopy. However, at least one of the inclusions of Definition 10.1(i) fails, hence $\oplus$ fails to yield a quotient sum relating the spaces $\bar{D}_{q}(Y, X)$.

We can get around this in one crucial case.

ExAmple 10.8. The coefficient system $C\left(R^{\infty}\right)$ is directed by the full spaces $F\left(R^{\infty}, r+1\right)$ and the maps $\lambda_{r}$ just specified. The point is that $\lambda_{r}: F\left(R^{\infty}, r\right) \rightarrow$ $F\left(R^{\infty}, r+1\right)$ is clearly itself a $\Sigma_{r}$-equivariant homotopy equivalence since both spaces are $\Sigma_{r}$-free and contractible. With these choices, the inclusions of Definition 10.1(i) hold trivially and the internal sum on $e\left(R^{\infty}\right)$ is weakly directed.

For a connected based space $X, \bar{C}\left(R^{\infty}, X\right)$ is homologically and, hence, stably equivalent to $Q_{0}\left(X^{+}\right)$[7, 3.1]. If $X=B G$ for a topological monoid $G$, then $\bar{C}\left(R^{\infty}, B G\right)$ is a model for $B\left(\Sigma_{\infty} \int G\right)$, and $\bar{D}_{q}\left(R^{\infty}, B G\right)$ is a model for the cofibre of the natural map $B\left(\Sigma_{q-1} \int G\right) \rightarrow B\left(\Sigma_{q} \int G\right)$. This is the example relevant to Snaith's applications [23]. While we shall state the main theorem of this section in proper generality, we should admit that our only present application is to the internal sum on $\mathcal{C}\left(R^{\infty}\right)$.

TheOREM 10.9. Let $\oplus:(\mathcal{Q}, \Re) \rightarrow \mathcal{C}$ be a weakly directed sum, where $\mathcal{Q}, \Re$ and $\mathcal{C}$ are $\Sigma$-free. For based spaces $X$ and $Y$, the following is a weakly homotopy commutative diagram in the stable category in which the horizontal arrows are equivalences:

$$
\begin{aligned}
& \Sigma^{\infty}(\bar{A} X \times \bar{B} Y) \stackrel{\Sigma_{r>1} \Sigma_{p+q=r} \bar{j}_{p} \wedge \bar{j}_{q}}{\longrightarrow} \underset{r \geqslant 1}{\vee} \underset{p+q=r}{\vee} \Sigma^{\infty}\left(\bar{D}_{p}(\mathcal{Q}, X) \wedge \bar{D}_{q}(\Re, Y)\right) \\
& \Sigma^{\infty} \oplus \downarrow \quad \downarrow \bigvee_{r \geqslant 1} \bigvee_{p+q=r} \oplus \\
& \Sigma^{\infty} \bar{C}(X \wedge Y) \longrightarrow \Sigma_{r>1} \bar{j}_{r}
\end{aligned}
$$


Proof. Crossing with $\bigodot_{\infty}$ as in the proof of Theorem 4.6 fails here since $\bigodot_{\infty}$ is not directed $[7,1.6]$. However, crossing with $\varrho\left(R^{\infty}\right)$ shows that we may assume, without loss of generality, that the given sum is weakly separated. The result follows as in the proof of [7, 2.7] and Theorem 4.6.

We close with two examples related to the classical James maps [9] and the James maps of Barratt and Eccles [3]. We leave the details to the interested reader.

EXERCISE 10.10. Let $\Re$ be the operad with $\Re_{j}=\Sigma_{j}$. The associated functor $M X$ on spaces is the James construction. Then $\Re$ is a directed coefficient system [7, 1.3] and admits James systems $\left\{\xi_{q, r}\right\}: \Re \rightarrow \mathfrak{N}$ for all $q[6,4.3]$. Verify that the block sum of permutations $\oplus:(\mathfrak{N}, \mathfrak{N}) \rightarrow \mathfrak{N}$ is directed. Show that, with all coefficient systems taken to be $\Re$ and with $\square$ defined in terms of $\oplus$ and the usual product $\otimes$, the diagram of Definition 3.3 would commute if the permutation $\tau_{r, u, v}$ were replaced by the identity; compare Remark 3.2 .

EXERCISE 10.11. By $[15, \S 10$ or $3, \mathrm{I}]$, there is a product-preserving functor from spaces to contractible spaces. Application of this functor to $\mathfrak{T}$ (that is, to symmetric groups) gives an operad $\mathscr{D}[15, \mathrm{p} .161]$. Verify that $\mathscr{D}$ inherits from $\mathscr{N}$ a structure of directed coefficient system, a directed sum $\oplus$, a product $\otimes$, and James systems $\left\{\xi_{q, r}\right\}: \mathscr{D} \rightarrow \mathscr{D}$ (compare [3, III]). Again, with all coefficient systems taken to be $\mathscr{D}$, the diagram of Definition 3.3 would commute if $\tau_{r, u, v}$ were replaced by the identity.

These examples give force to the sentence above Proposition 3.4.

Appendix. Good embeddings. Here we prove generalizations of Lemmas 2.6 and 5.5. The following is a start. We write $e_{i}$ for the $i$ th restriction of an embedding $e$ : $\mathrm{II}_{1 \leqslant i \leqslant r} Y \rightarrow Z$.

Lemma A.1. Let $e: \amalg_{1 \leqslant i \leqslant r} Y \rightarrow Z$ and $f: \amalg_{1 \leqslant i \leqslant r} Z \rightarrow Z$ be embeddings. Assume that the following conditions hold for each $i$ :

(i) There is an isotopy $E_{i}: e_{i} \simeq j_{i}$ for some chosen embedding $j_{i}$.

(ii) There is an isotopy $F_{i}: f_{i} \simeq 1$ with $f_{i}(Z) \subset F_{i, t}(Z), 0 \leqslant t \leqslant 1$.

(iii) $e_{i}(Y)$ is contained in $f_{i}(Z)$.

Then $e$ and $f \circ\left(\amalg_{1 \leqslant i \leqslant r} j_{i}\right)$ are isotopic.

Proof. The unique maps $G_{i, t}: Y \rightarrow Y$ such that $f_{i, t} \circ G_{i, t}=e_{i}$ specify an isotopy. An isotopy $H: e \simeq f \circ\left(\amalg_{1<i \leqslant r} j\right)$ is obtained by letting its $i$ th restriction begin by traversing $f_{i} \circ G_{i}: e_{i} \simeq f_{i} \circ e_{i}$ and end by traversing $f_{i} \circ E_{i}: f_{i} \circ e_{i} \simeq f_{i} \circ j$.

Lemma 2.6 is a special case of the following result. Note that if $m<n$, then any embedding $R^{m} \rightarrow R^{n}$ obtained as a composite of permutations and diagonal maps on certain coordinates is isotopic to the standard inclusion.

LEMMA A.2. Let $m \leqslant n$, where $m \geqslant 1$ and $n \geqslant 2$. Then any two embeddings $e: \amalg_{1 \leqslant i \leqslant r} R^{m} \rightarrow R^{n}$ such that, for each $i, e_{i}$ is isotopic to some fixed chosen embedding $j_{i}$ are isotopic.

Proof. It suffices to prove the result for $e$ and $f \circ\left(\amalg_{1 \leqslant i \leqslant r} j_{r}\right)$, where $f$ is a conveniently chosen fixed good embedding. Choose $r$ distinct points $y_{i}$ and choose $f_{i}$ : $R^{n} \rightarrow R^{n}$ such that $f_{i}(0)=y_{i}$, the images of the $f_{i}$ are disjoint, and there are isotopies 
$F_{i}: f_{i} \simeq 1$ with $f_{i}\left(R^{n}\right) \subset F_{i, t}\left(R^{n}\right), 0 \leqslant t \leqslant 1$. By the previous lemma, it suffices to prove that $e$ is isotopic to $e^{\prime}$, where $e_{i}^{\prime}\left(R^{m}\right)$ is contained in $f_{i}\left(R^{n}\right)$. Choose points $x_{i} \neq y_{i}$ in the image of $e_{i}$. Since $n \geqslant 2$, we can choose embedded nonintersecting paths $\lambda_{i}$ from $x_{i}$ to $y_{i}$. (It is only here that we use $n \geqslant 2$; if $m=n=1$, there is a unique $\tau \in \Sigma_{r}$ such that $e \tau$ is isotopic to $f$, where $(e \tau)_{i}=e_{\tau(i)}$.) Construct an isotopy $H$ of $R^{n}$ such that $H_{t}\left(x_{i}\right)=\lambda_{i}(t)$ for $1 \leqslant i \leqslant r$ and $0 \leqslant t \leqslant 1$. We can choose $\varepsilon>0$ so that the ball $B_{\varepsilon}\left(x_{i}\right)$ of radius $\varepsilon$ around $x_{i}$ is carried into the image of $e_{i}$ by $H_{1}$, and so that $H_{t} B_{\varepsilon}\left(x_{i}\right)$ and $H_{t} B_{\varepsilon}\left(x_{j}\right)$ have empty intersection for $i \neq j$ and all $t$. Clearly we may apply a shrinking isotopy to arrange that each $e_{i}$ carries all of $R^{m}$ into $B_{\varepsilon}\left(x_{i}\right)$. Then $e^{\prime}=H_{1} \circ e$ is as required.

Turning towards Lemma 5.5, consider $R^{n} \times Y$. A good embedding $i: R^{n} \amalg R^{n} \rightarrow R^{n}$ induces the good embedding $\left(R^{n} \times Y\right) \amalg\left(R^{n} \times Y\right) \rightarrow R^{n} \times Y$ with restrictions $i_{k} \times$ 1. If $n \geqslant 2$, the induced $H$-space structure on $C\left(R^{n} \times Y, X\right)$ is independent of the choice of $i$. By the parenthetical remark in the above proof, there are two potentially different products if $n=1$ and they agree if and only if either is homotopy commutative. Let

$$
e(i): B\left(R^{n} \times Y, q\right) \amalg B\left(R^{n} \times Y, q\right) \rightarrow B\left(R^{n} \times Y, q\right)
$$

be the embedding with restrictions $B\left(i_{k} \times 1, q\right)$. Observe next that the map $\pi: B\left(R^{n} \times Y, q\right) \rightarrow R^{n}$, defined by

$$
\pi\left\langle\left(v_{1}, y_{1}\right), \ldots,\left(v_{q}, y_{q}\right)\right\rangle=\frac{1}{q}\left(v_{1}+\cdots+v_{q}\right),
$$

is $R^{n}$-equivariant, where $R^{n}$ acts by coordinatewise addition on $B\left(R^{n} \times Y, q\right)$ and by addition on $R^{n}$. Therefore $B\left(R^{n} \times Y, q\right)$ is homeomorphic to $R^{n} \times Z$, where $Z=$ $\pi^{-1}(0)$. For $Y$ a point, we identified $Z$ as $F\left(R^{n}-0, q-1\right) / \Sigma_{q}$ in $[6,5.7]$. Let

$$
f(i): B\left(R^{n} \times Y, q\right) \amalg B\left(R^{n} \times Y, q\right) \rightarrow B\left(R^{n} \times Y, q\right)
$$

be the embedding with restrictions $i_{k} \times 1$. With $Y$ a point, the following result specializes to Lemma 5.5.

LEMMA A.3. The good embeddings $e(i)$ and $f(i)$ above are isotopic.

Proof. If $n \geqslant 2$, we need only prove this for any one chosen $i$. If $n=1$, we need only consider one $i$ in each of the two inequivalent classes of good embeddings $R \amalg R \rightarrow R$. In any case, we may assume that there are isotopies $I_{k}: i_{k} \simeq 1$ such that $i_{k}\left(R^{n}\right) \subset I_{k, t}\left(R^{n}\right)$ for all $t$. The $I_{k}$ induce analogous isotopies $E_{k}: e(i)_{k} \simeq 1$ and $F_{k}$ : $f(i)_{k} \simeq 1$. An easy calculation shows that the image of $e(i)_{k}$ is contained in the image of $f(i)_{k}$ provided that each average $\frac{1}{q}\left(i_{k}\left(v_{1}\right)+\cdots+i_{k}\left(v_{q}\right)\right)$ has the form $i_{k}(w)$ for some $w$. This holds if we take $i_{k}=h_{k} \times 1$, where $h_{k}: R \rightarrow R$ is an increasing embedding with image either $(0, \infty)$ or $(\infty, 0)$. Lemma A.1 then applies to give the conclusion.

We conclude with an amusing exercise in these techniques.

LemMA A.4. Let $i: Y \amalg Y \rightarrow Y$ and $j: Z \amalg Z \rightarrow Z$ be good embeddings. Then the good embeddings $(Y \times Z) \amalg(Y \times Z) \rightarrow Y \times Z$ induced by $i$ and $j$ are isotopic. 


\section{BIBLIOGRAPHY}

1. J. F. Adams, The Kahn-Priddy theorem, Math. Proc. Cambridge Philos. Soc. 73 (1973), 45-55.

2. Infinite loop spaces, Princeton Univ. Press, Princeton, N.J., 1978.

3. M. G. Barratt and P. J. Eccles, $\Gamma^{+}$-structures. I-III, Topology 13 (1974), 25-45, 113-126, 199-207.

4. J. Caruso, Configuration spaces and mapping spaces, Thesis, Univ. of Chicago, 1979.

5. F. R. Cohen, T. Lada and J. P. May, The homology of iterated loop spaces, Lecture Notes in Math., vol. 533, Springer-Verlag, Berlin and New York, 1976.

6. F. R. Cohen, J. P. May and L. R. Taylor, Splitting of certain spaces $C \underline{X}$, Math. Proc. Cambridge Philos. Soc. 84 (1978), 465-496.

7. __ Splitting of some more spaces, Math. Proc. Cambridge Philos. Soc. 86 (1979), 227-236.

8. $K(Z, 0)$ and $K\left(Z_{2}, 0\right)$ as Thom spectra, Illinois J. Math. 25 (1981), 99-106.

9. I. M. James, Reduced product spaces, Ann. of Math. (2) 62 (1955), 170-197.

10. D. S. Kahn and S. B. Priddy, The transfer and stable homotopy theory, Math. Proc. Cambridge Philos. Soc. 83 (1978), 103-111.

11. U. Koschorke and B. Sanderson, Self intersections and higher Hopf invariants, Topology 17 (1978), 283-290.

12. N. J. Kuhn, The geometry of the James-Hopf maps, Pacific J. Math. 102 (1982), 397-412.

13. L. G. Lewis, When is the natural map $X \rightarrow \Omega \Sigma X$ a cofibration?, Trans. Amer. Math. Soc. 273 (1982), 147-156.

14. P. Löffler and N. Ray, A geometric proof of a theorem of Kahn and Priddy, preprint.

15. M. Mahowald, Ring spectra which are Thom complexes, Duke Math. J. 49 (1979), 549-559.

16. J. P. May, The geometry of iterated loop spaces, Lecture Notes in Math., vol. 271, Springer-Verlag, Berlin and New York, 1972.

17. $E_{\infty}$ spaces, group completions, and permutative categories, London Math. Soc. Lecture Note Ser., no. 11, Cambridge Univ. Press, Cambridge, 1974, pp. 61-93.

18. Applications and generalizations of the approximation theorem, Lecture Notes in Math., vol. 763, Springer-Verlag, Berlin and New York, 1979, pp. 38-69.

19. J. Milnor, On spaces having the homotopy type of a CW-complex, Trans. Amer. Math. Soc. 90 (1959), $272-280$.

20. G. Segal, Configuration spaces and iterated loop spaces, Invent. Math. 21 (1973), 213-221.

21. Operations in stable homotopy theory, London Math. Soc. Lecture Note Ser., no. 11, Cambridge Univ. Press, Cambridge, 1974, pp. 105-110.

22. V. P. Snaith, $A$ stable decomposition for $\Omega^{n} \Sigma^{n} X$, J. London Math. Soc. (2) 7 (1974), 577-583.

23. __ Localized stable homotopy of some classifying spaces, Math. Proc. Cambridge Philos. Soc. 89 (1981), 325-330.

ATEX CORPORATION, BEDFORD, MASSACHUSETTS 01730

Department of MAThematics, University of KentuCKy, LeXington, KentuCKy 40506

Department of Mathematics, University of Chicago, Chicago, Illinois 60637

Department of Mathematics, University of Notre Dame, Notre Dame, Indiana 46556 